\title{
CRISPR-Cas Systems in Bacteroides fragilis, an Important Pathobiont in the Human Gut Microbiome
}

\author{
Mehrdad Tajkarimi ${ }^{1}$ and Hannah M. Wexler ${ }^{1,2,3 *}$ \\ ${ }^{1}$ Brentwood Biomedical Research Institute, Los Angeles, CA, United States, ${ }^{2}$ University of California, Los Angeles, Los \\ Angeles, CA, United States, ${ }^{3}$ GLAVA Health Care System, Los Angeles, CA, United States
}

\section{OPEN ACCESS}

Edited by:

Scott Norman Peterson

Sanford-Burnham Institute for Medical

Research, United States

Reviewed by:

Andrei Osterman,

Sanford Burnham Prebys Medical

Discovery Institute (SBP),

United States

Jozsef Soki,

University of Szeged, Hungary

*Correspondence: Hannah M. Wexler

hwexler@ucla.edu

Specialty section: This article was submitted to

Evolutionary and Genomic

Microbiology

a section of the journa

Frontiers in Microbiology

Received: 23 July 2017 Accepted: 31 October 2017 Published: 23 November 2017

Citation:

Tajkarimi M and Wexler HM (2017) CRISPR-Cas Systems in Bacteroides fragilis, an Important Pathobiont in the Human Gut Microbiome.

Front. Microbiol. 8:2234.

doi: 10.3389/fmicb.2017.02234
Background: While CRISPR-Cas systems have been identified in bacteria from a wide variety of ecological niches, there are no studies to describe CRISPR-Cas elements in Bacteroides species, the most prevalent anaerobic bacteria in the lower intestinal tract. Microbes of the genus Bacteroides make up 25\% of the total gut microbiome. Bacteroides fragilis comprises only $2 \%$ of the total Bacteroides in the gut, yet causes of $>70 \%$ of Bacteroides infections. The factors causing it to transition from benign resident of the gut microbiome to virulent pathogen are not well understood, but a combination of horizontal gene transfer (HGT) of virulence genes and differential transcription of endogenous genes are clearly involved. The CRISPR-Cas system is a multi-functional system described in prokaryotes that may be involved in control both of HGT and of gene regulation.

Results: Clustered regularly interspaced short palindromic repeats (CRISPR) elements in all strains of $B$. fragilis $(n=109)$ with publically available genomes were identified. Three different CRISPR-Cas types, corresponding most closely to Type IB, Type IIIB, and Type IIC, were identified. Thirty-five strains had two CRISPR-Cas types, and three strains included all three CRISPR-Cas types in their respective genomes. The cas 1 gene in the Type IIIB system encoded a reverse-transcriptase/Cas1 fusion protein rarely found in prokaryotes. We identified a short CRISPR (3 DR) with no associated cas genes present in most of the isolates; these CRISPRs were found immediately upstream of a hipA/hipB operon and we speculate that this element may be involved in regulation of this operon related to formation of persister cells during antimicrobial exposure. Also, blood isolates of $B$. fragilis did not have Type IIC CRISPR-Cas systems and had atypical Type IIIB CRISPR-Cas systems that were lacking adjacent cas genes.

Conclusions: This is the first systematic report of CRISPR-Cas systems in a wide range of $B$. fragilis strains from a variety of sources. There are four apparent CRISPRCas systems in $B$. fragilis - three systems have adjacent cas genes. Understanding CRISPR/Cas function in $B$. fragilis will elucidate their role in gene expression, DNA repair and ability to survive exposure to antibiotics. Also, based on their unique CRISPR-Cas arrays, their phylogenetic clustering and their virulence potential, we are proposing that blood isolates of $B$. fragilis be viewed a separate subgroup.

Keywords: Bacteroides, virulence, CRISPR-Cas system, phage, immune defense, gut microbiome, pathobiome 


\section{INTRODUCTION}

Bacteria are constantly exposed to incoming DNA via phages, plasmids, other mobile genetic elements (MGEs) or "naked" nucleic acid from lysed cells. Their ability to incorporate these bits of DNA into their own genetic code, known as horizontal gene transfer (HGT), contributes to the ability of a bacterium to adapt to a wide variety of ecological and environmental pressures, including antibiotics and evolving host niche. Along with this ability to incorporate DNA, bacteria possess immune defense mechanisms to defend themselves against invading DNA or RNA in their immediate environment, including systems to abort infection, specialized enzymes that degrade foreign DNA (restriction/modification or R/M systems), and an adaptive immunity system first described more than a decade ago (Haft et al., 2005) that confers adaptive immunity to invading DNA or RNA (Marraffini, 2015). This system, the CRISPR-Cas system, is defined by clustered regularly interspaced short palindromic repeats (CRISPR) and the CRISPR-associated (Cas) proteins (Haft et al., 2005).

The specificity of the CRISPR system lies in the short, repetitive (sometimes palindromic) direct repeat sequences (DR) separated by nucleic acid sequences (spacers) previously acquired from invading DNA and a cleavage system that can target incoming DNA based on recognition of those previously encountered DNA sequences (Burstein et al., 2016). The Cas protein machinery that mediates the protection against invading nucleic acid is encoded by gene clusters that are adjacent to and generally upstream of the CRISPR locus (Jore et al., 2011). The CRISPR RNA array (crRNA) that includes the entire DR sequence with the intervening spacers is transcribed (from the leader) as a single transcript and later processed into individual segments that contain one spacer sequence and portions of the DR sequence at both ends. These individual crRNAs are eventually used to direct Cas proteins to destroy incoming invasive genetic elements (including phages, plasmids and other nucleic acid segments) that include sequences complementary to one of the spacer sequences in the CRISPR. Newly acquired spacers are incorporated into CRISPR loci in a directional manner, generally, but not invariably, at repeats adjacent to CRISPR leaders (Rath et al., 2015).

Many surveys reported that a vast number of prokaryotic species possess CRISPR arrays (Louwen et al., 2014; including $\sim 45 \%$ of bacteria and $\sim 83 \%$ of Archaea; Grissa et al., 2007) although there are entire phyla that lack this system (Burstein et al., 2016). CRISPR-Cas systems were classified into 18 structural families and 24 sequence families (Makarova et al., 2015). While new subtypes are still being identified, the major groups include 5 major types and 16 different subtypes. The basis for these classifications include which cas genes are present, the cas operon architecture, Cas protein sequences, and the nuclease involved in the degradation of the target DNA (Barrangou, 2015). The function of some of the cas genes has been described in detail, while the function(s) of other genes remains unknown (Rath et al., 2015). The most common systems described thus far in prokaryotes are variants of the (1) Class 1 Type I system, (2) the Class 1 Type III system, and (3) the Class 2 Type II system
(Makarova et al., 2015). In general, most strains of the same species contain identical CRISPR-Cas types (Louwen et al., 2014) although there are some variations.

Since the host CRISPR/Cas can acquire and incorporate incoming DNA as spacer DNA, the resultant CRISPR may serve as a molecular record of past exposure to foreign DNA for an individual strain. Alternatively, entire CRISPR loci may be disseminated via HGT There is evidence that these systems are readily transferred between microbes, potentially even across phylum boundaries (Burstein et al., 2016); this is supported both by the phylogenetic relationship of the Cas genes from diverse organisms (Godde and Bickerton, 2006) as well as the fact that DR sequences from diverse organisms can be clustered together into subtypes based on sequence similarities (Kunin et al., 2007).

The function of the CRISPR-Cas system was initially described as protection against invasive nucleic acid. However, the sheer diversity of the systems suggested other functions, and indeed, these elements have now been implicated in regulation of transcription, chromosomal segregation and rearrangement and DNA repair, although most of the processes related to these alternate functions are not completely clear at this point (Stern et al., 2010; Sampson and Weiss, 2014). The potential of regulation via suppressing transcription is also suggested by the broad use of modified Cas9 nuclease precisely to suppress transcription in engineered systems (Barrangou et al., 2007; Sander and Joung, 2014; Mimee et al., 2015; Mougiakos et al., 2016).

The presence of CRISPR-Cas systems are variably associated with virulence (Makarova et al., 2011; Barrangou, 2015) and/or antibiotic resistance in pathogenic bacteria. For example, the presence of a CRISP-Cas locus correlated inversely with acquired antibiotic resistance (Palmer and Gilmore, 2010) in Enterococcus faecalis; a mechanism for CRISPR-Cas loss in this species was identified and the data suggested that antibiotic use inadvertently selects for enterococcal strains with compromised genome defense. Conversely, an E. faecalis strain harboring CRISPR-Cas was more virulent than one lacking this element (Bourgogne et al., 2008). The type of CRISPR element may also be related to the virulence of the bacterium: CRISPR typing was able to discriminate between lineages of Propionibacterium acnes with varying degrees of virulence although there were strains belonging to the more invasive lineages that lacked such spacers or a complete CRISPR-Cas system (Marinelli et al., 2012). On the one hand, the ability of a bacterium to incorporate mobile elements bearing pathogenic determinants would argue for a less robust CRISPR-Cas defense system; on the other hand, the presence of multiple CRISPRs systems with a variety of spacers that may indicate previous exposure to these elements, parts of which were incorporated into the bacterial genome.

While CRISPR-Cas systems were identified in bacteria from a wide variety of ecological niches, there are no studies describing CRISPR-Cas elements in Bacteroides species, the most prevalent anaerobic bacteria in the lower intestinal tract. The species Bacteroides fragilis $(\mathrm{BF})$, an important gut pathobiont, is of particular interest, since its transition from friendly commensal to dangerous threat is not well understood. Outside its colonic niche, $B$. fragilis is an opportunistic pathogen; $B$. fragilis only 
accounts for $2 \%$ of the total gut Bacteroides yet it is the agent of $>70 \%$ of Bacteroides infections. B. fragilis is the main cause of anaerobic bacteremia and intraabdominal abscesses, implicated in serious gynecological, soft tissue infections, peritonitis, brain abscess (Wexler, 2014) and surgical site infections (SSIs) following colorectal surgery (Solomkin et al., 2010). Even $B$. fragilis strains isolated as normal gut microbiota possess many genes associated with virulence; as the expression of virulent genes constitutes a form of stress response of pathogenic bacteria during host infection, we would expect differential regulation of the multitude of genes involved in virulence, survival, and host colonization (Louwen et al., 2014).

Bacteroides fragilis are also important players in the "hot spot" of HGT between microbes (Kurokawa et al., 2007) and constitute one of the most concentrated reservoirs of resistance genes in the human gut (Salyers et al., 2004). HGT throughout diverse bacterial species has been responsible for the dissemination of both virulence and resistance genes that undermine the usefulness of most antimicrobials (Barlow, 2009). In this gut milieu, resistance genes can move from commensal organisms to potential pathogens, between pathogens, and even between pathogens and probiotics (Capozzi and Spano, 2009). Genetic analysis of $B$. fragilis clinical isolates as well as isolates from the GI tract indicate a high degree of HGT, including resistance genes from very divergent gram-positive bacteria (Husain et al., 2014).

The importance of CRISPR-Cas systems in B. fragilis is of particular interest, given the potential of these systems to regulate and/or record HGT, including the acquisition of virulence and antimicrobial resistance genes. CRISPR-Cas systems may also regulate endogenous genes, some of which may be important in the transition of $B$. fragilis from commensal to virulent pathogen. The possible ability of Type I CRISPR-Cas to effect DNA repair would be highly significant for $B$. fragilis, which is very susceptible to the DNA-damaging properties of oxygen exposure. The aim of our study was to investigate and document the occurrence, prevalence and diversity of CRISPR-Cas systems in B. fragilis strains with publically available genomes, including strains from a variety of clinical sources and belonging to different phylogenetic clades. With this information, we will explore the capacity of the naturallyoccurring CRISPR-Cas systems to regulate gene expression, control HGT (including transmission of antimicrobial resistance and virulence determinants) and control reaction to antibiotic exposure.

\section{METHODS}

\section{Strains}

One hundred nine published sequences of $B$. fragilis as well as the complete genome sequence of $B$. fragilis species UW (a blood isolate reported which clusters phylogenetically with a subset of strains from B. fragilis; Salipante et al., 2015) were retrieved from the National Center for Biotechnology Information (NCBI, http://www.ncbi.nlm.nih.gov/genome). The assembly numbers of these complete genomes, the common names of the strains, and the type of infection from which they were isolated are listed in Table 1. Some strains were classified as virulent or multidrug resistant without the source of isolation; these are so noted.

\section{Phylogenetic Analysis of $B$. fragilis Strains}

The J species Web Server (with the kind assistance of Dr. Michael Richter, Ribocon, Bremen, Germany) was used to construct a matrix of average nucleotide identity between two organisms (ANIb) values among the $B$. fragilis strains tested. The matrix was converted to a three column array using an Excel macro and then converted into a Newick tree file using a routine in $\mathrm{R}$ (with the kind assistance of Dr. Luis M. Rodriguez, Kostas Lab, Georgia Institute of Technology, Atlanta, GA). The Newick file was visualized in the Archaeopteryx software tool (Han and Zmasek, 2009).

\section{Identification of CRISPRs in B. fragilis}

CRISPR arrays were identified using both the CRT tool (Bland et al., 2007) with default parameters and the CRISPR Repeat Finder program (Grissa et al., 2007). We found the CRT program to be generally more robust than CRISPR Finder in detecting CRISPRs; on the other hand, CRT does not have a filter to limit acceptable spacer homology within a given CRISPRs, leading to incorrectly identifying tandem repeat sequences (perfect and imperfect) as CRISPRs. The CRT program was used with default parameters and the CRISPR Repeat Finder program was used with the following parameters: Min Number of Repeats 2, Min Repeat Length 19, Max Repeat Length 55, Search Window 8, Min Spacer length 18 and Max Spacer Length 55. CRISPRS repeats that had three or more DR sequences were retained for further analysis and then validated using the CRISPTionary program (Grissa et al., 2007).

\section{Distribution of Spacers among CRISPRs}

The CRISPtionary tool (Grissa et al., 2007) was used to analyze spacer distribution and position with individual CRISPRs and to construct a binary file of spacer distribution among the various CRISPR-Cas systems.

\section{Identification of Protospacer Target Regions}

Clustered regularly interspaced short palindromic repeats (CRISPR) targets were identified by submitting the spacer sequences to analysis with the CRISPR Target tool (http:// bioanalysis.otago.ac.nz/CRISPRTarget) searching the GenBank Phage, RefSeq Bacteria, RefSeq plasmid and RefSeq viral databases. We also searched the Nucleotide collection (nr/nt) at NCBI using the default parameters, both with and without the limit of "NOT Repeat_Region" added as a query filter. Each matched protospacer was then subjected to a BLAST search (Benson et al., 2014) to find full or partial matches to any other phage, bacteria, plasmid and viruses; annotation of the ten up- and downstream genes flanking the proposed match were recorded. 
TABLE 1 | Bacteroides fragilis strains used in this study.

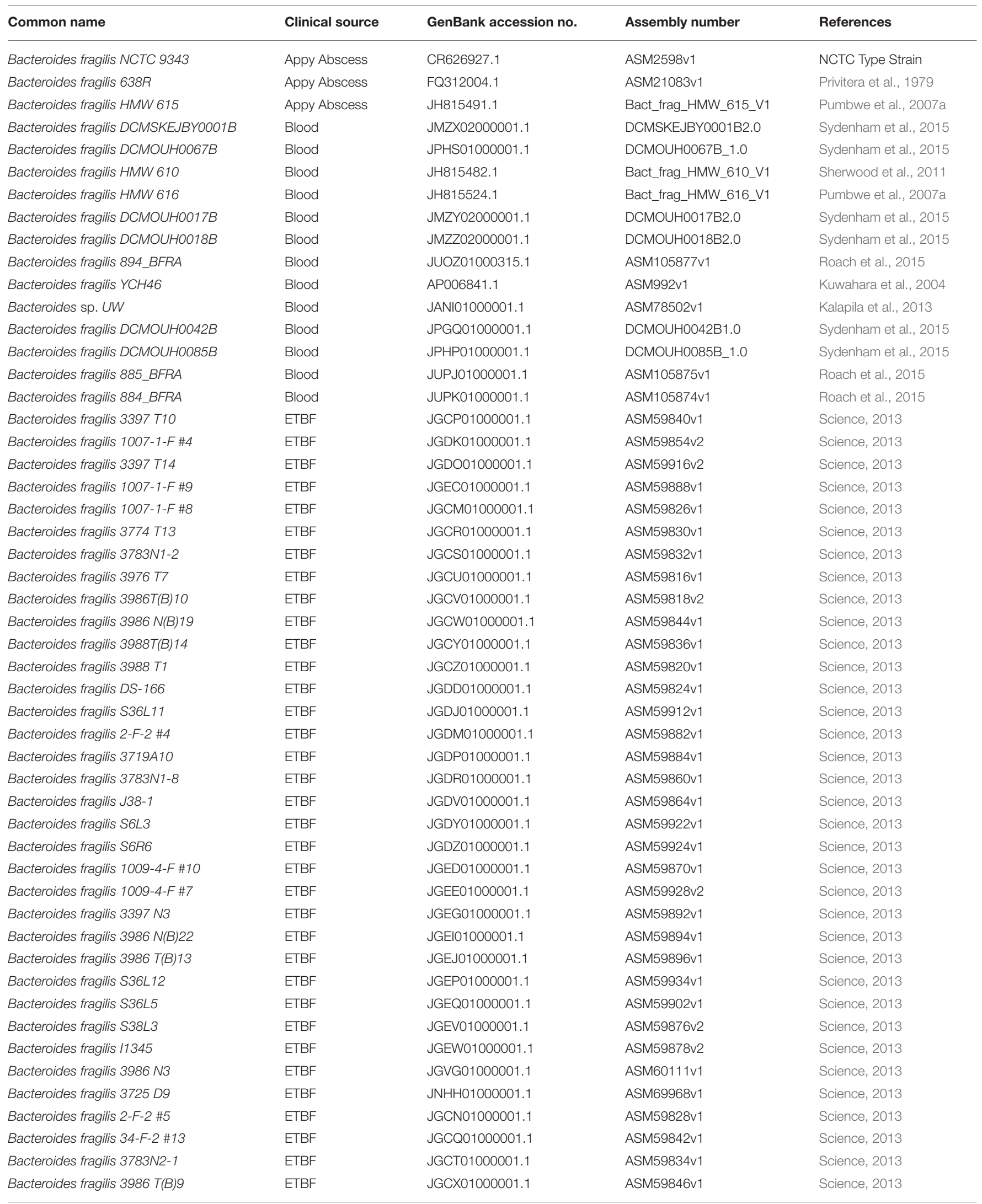


TABLE 1 | Continued

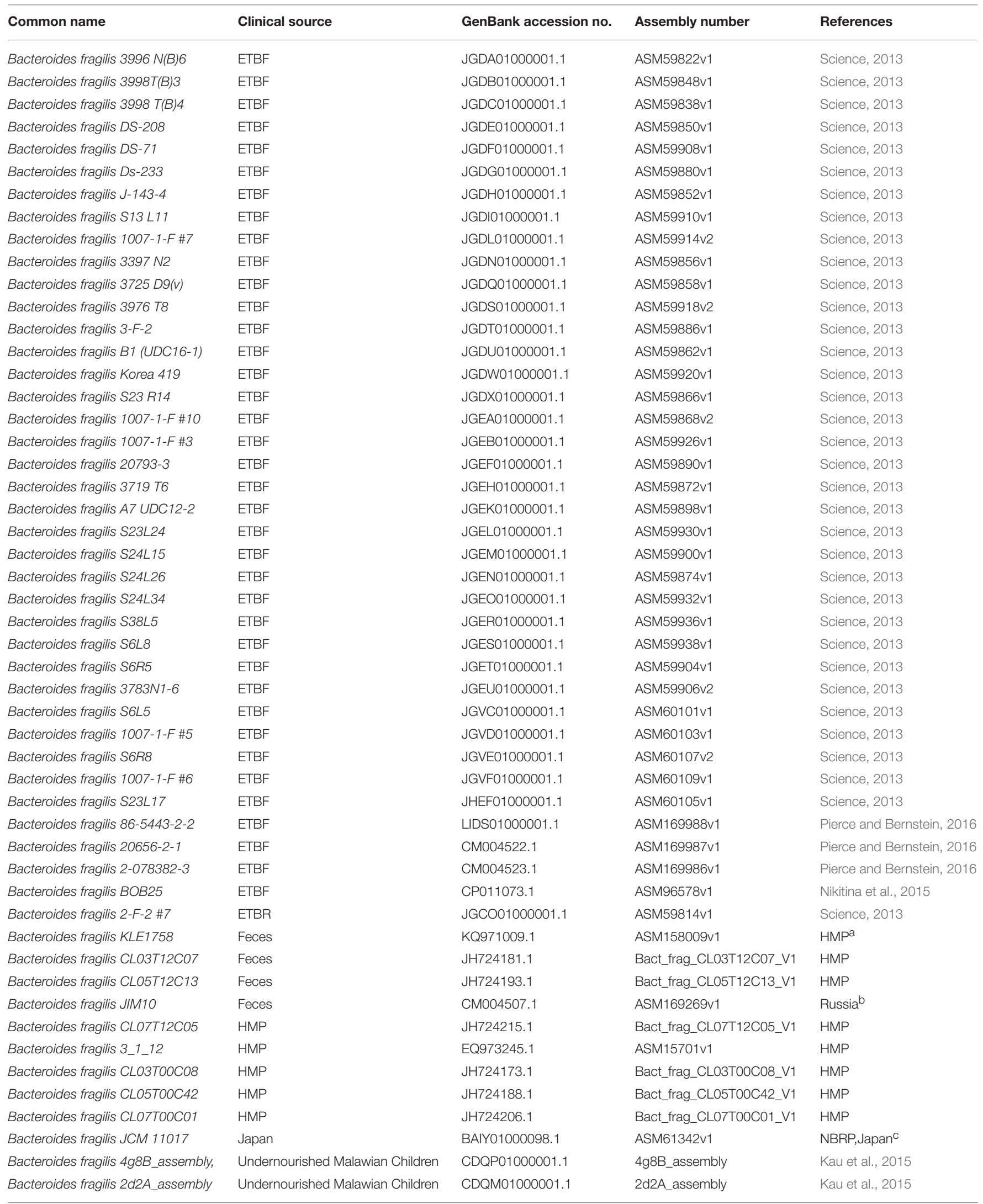


TABLE 1 | Continued

\begin{tabular}{|c|c|c|c|c|}
\hline Common name & Clinical source & GenBank accession no. & Assembly number & References \\
\hline Bacteroides fragilis BF8 & ViR/MDR & LGTH01000001.1 & ASM169535v1 & Soki et al., 2016 \\
\hline Bacteroides fragilis S14 & ViR/MDR & CP012706.1 & ASM168221v1 & Sokid \\
\hline Bacteroides fragilis 322_BFRA & Fluid & JVLP01000024.1 & ASM105489v1 & Roach et al., 2015 \\
\hline Bacteroides fragilis 321 BFRA & Fluid & JVLQ01000008.1 & ASM105633v1 & Roach et al., 2015 \\
\hline Bacteroides fragilis 320_BFRA & Fluid & JVLR01000007.1 & ASM105486v1 & Roach et al., 2015 \\
\hline Bacteroides fragilis $0: 21$ & $\mathrm{ViR} / \mathrm{MDR}$ & KV751174.1 & ASM169369v1 & Soki et al., 2016 \\
\hline Bacteroides fragilis BFBE1.1 & ViR/MDR & LN877293.1 & BFBE1.1 & Risse et al., 2015 \\
\hline
\end{tabular}

${ }^{a}$ Reference Genome for the Human Microbiome Project (HMP).

${ }^{b}$ Federal Research and Clinical Center of Physical-Chemical Medicine of FMBA of Russia.

${ }^{c}$ National Bioresearch Project, Japan.

${ }^{d}$ Soki et al. (unpublished), Comparative analysis of Division I and II Bacteroides fragilis strain genomes.

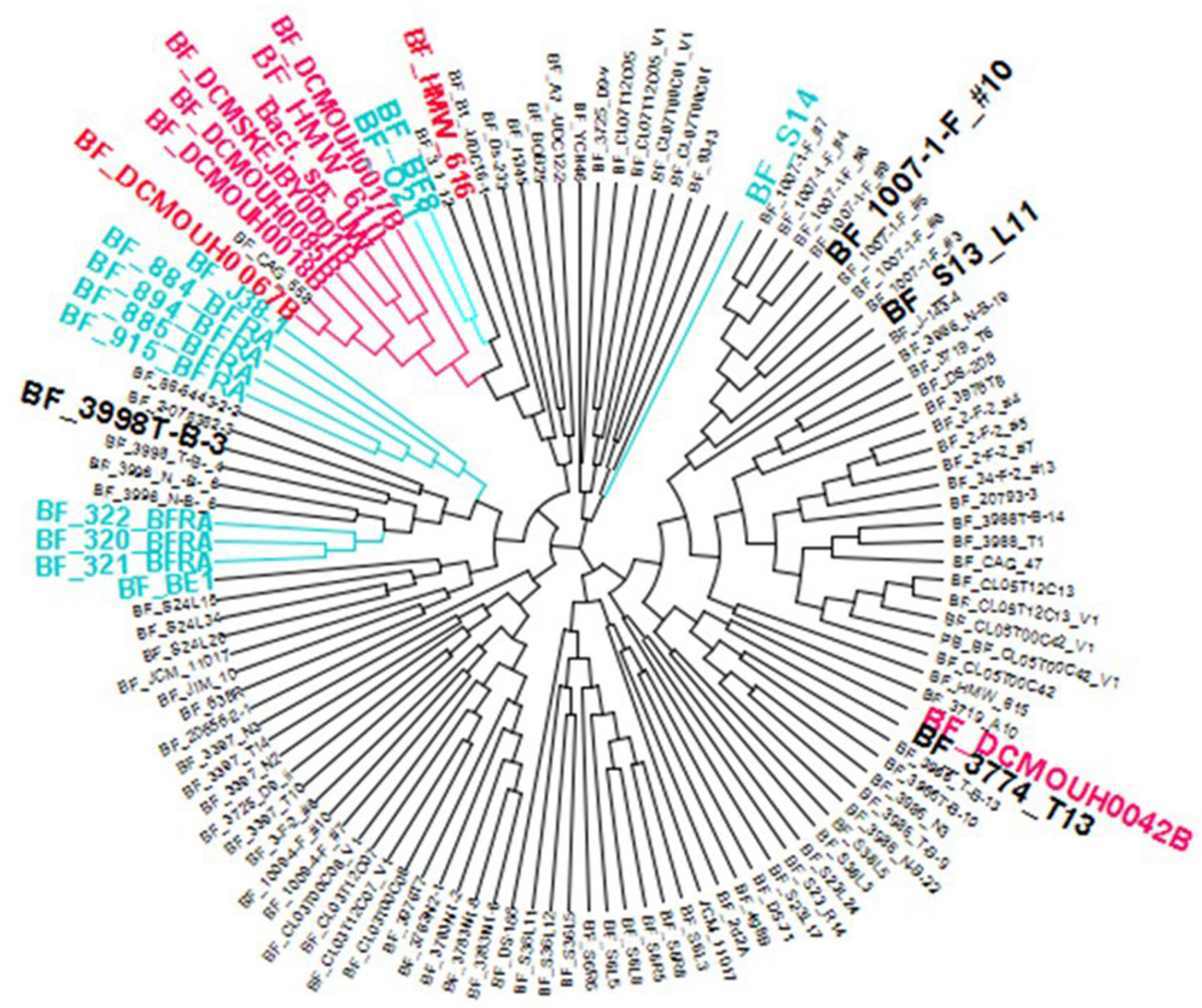

FIGURE 1 | Dendrogram of $B$. fragilis isolates. The dendrogram is based on ANIb values generated for published $B$. fragilis genomes. It is viewed using the Archaeopteryx software tool. B. fragilis strains isolated from blood are colored in red. (Although BF Cag 558 is clustered with the blood isolates, there is no available source data). All blood isolates (in red) are clustered together, apart from BF_DCMOUHOO42B (this isolate also has a CRISPR pattern distinct from the other isolates.) Isolates described as multidrug resistant or virulent (teal) are more widely scattered. ETBF isolates are scattered across the dendrogram. Several of the strains are emphasized in a larger black font because their spacer distribution was of particular interest (discussed in Figure 7). 


\section{Identification of cas Genes Associated with Particular CRISPR Repeats}

Genes flanking specific CRISPR loci were identified by BLAST analysis of the entire CRISPR sequence against the host genome using RAST-based annotation (Aziz et al., 2008). We have found that the RAST annotation server frequently offered a more robust annotation than GenBank and in this study it afforded an easier way to localize the CRISPR element and to view the adjacent genes. Therefore, all bacterial genomes were uploaded to the RAST server and the adjacent cas genes as well as the genomic context of the particular CRISPR repeat array were determined. In some cases, the CRISPR was at the end of a gene scaffold, and the upstream genes could not be identified. The particular cas operon identified was assigned to a CRISPR-Cas classification system developed by Makarova (Makarova et al., 2011). Most of the $B$. fragilis genome sequences have not been completely assembled, and sometimes the CRISPR repeat sequence was located near the edge of a contig; in those cases, the adjacent genes could not be identified. In those cases, the presence or absence of cas genes somewhere else on the genome was noted. Automatic assembly of contigs for genome assembly is often complicated by the presence of transposase genes, and indeed, we found transposase genes adjacent to the CRISPR repeat element in many instances.

\section{Other Bioinformatic Tools Used in the Analysis of $B$. fragilis CRISPRs}

Consensus sequences and images for the DR sequences were obtained using WEBLOGO (Crooks et al., 2004). Predicted structures of the DR repeat sequence were visualized using the RNA fold program (Ding et al., 2005). Phylogenetic relationships of the consensus DR sequences in the DR database were analyzed using CRISPRmap (Lange et al., 2013). Gene neighborhoods were visualized using tools at the Joint Genome Institute (Markowitz et al., 2012). Venn diagrams were generated using http:// bioinformatics.psb.ugent.be/webtools/Venn/.

\section{RESULTS}

\section{All B. fragilis Strains with Publically Available Genome Sequences Were Analyzed for CRISPR Repeat Arrays}

Deep sequence analysis of the CRISPR system was performed for all of the publicly available genome sequences of $B$. fragilis $(n=$ 109) (Table 1). The proportion of "pathogenic" vs. "commensal" strains of $B$. fragilis is artificially inflated, since strains from serious infections are more likely to be chosen for whole genome sequencing. A large proportion of these strains are from studies of enterotoxigenic $B$. fragilis (ETBF), so these strains were disproportionately represented and consequently analyzed. Conversely, only a few strains from "normal" feces or human microbiome projects (where samples are often from healthy patients) have been sequenced.

The B. fragilis strains included in our study include several blood isolates from geographically distant locations including Washington State (Schapiro et al., 2004), Seattle (Kalapila et al., 2013), Denmark (Ank et al., 2015), the United Kingdom (Pumbwe et al., 2007b), and Afghanistan (Sherwood et al., 2011). A previous phylogenetic analysis of several clinical $B$. fragilis isolates indicated that there is a cluster of $B$. fragilis strains that constitute a genomospecies distinct from other $B$. fragilis (Salipante et al., 2015). Two of these isolates, BF HMW 610 and BF HMW 616, were reported and characterized extensively in our laboratory (Pumbwe et al., 2007a,b; Sherwood et al., 2011). The isolates noted as virulent and multidrug resistant (VIR/MDR) include an international cluster of MDR B. fragilis isolates from five countries (Soki et al., 2016). Whole genome sequencing of a large number of ETBF isolates has been undertaken and these are also included (Science, 2013). References, when available, for all of the isolates analyzed are listed in Table $\mathbf{1}$, and include more details about the origin and nature of these isolates.

\section{Phylogenetic Grouping of $B$. fragilis Strains}

A dendrogram based on Average Nucleotide Identity by BLAST (ANIb) values is shown in Figure 1. All but one of the strains isolated from blood cluster together in one group (despite being isolated in geographically distant areas.) Strains associated with enterotoxin related illnesses formed the majority of the strains and were evenly distributed across the tree as were "commensal" strains isolated as part of the human microbiome project.

\section{CRISPR-Cas Systems in B. fragilis}

Three types of CRISPR-Cas systems (and a fourth array without associated cas genes) are variably distributed in B. fragilis strains (Table 2) and most closely match types Class 1 Type IB, Class 1 Type IIIB, and Class 2 Type IIC (Makarova et al., 2015; Figure 2); each was in a highly conserved gene neighborhood (Figure 3). One hundred strains of $B$. fragilis from those studied had one or more CRISPR-Cas systems; 9 strains had no discernable CRISPRs (Figure 4). If the Orphan CRISPR-Cas system was excluded from the analysis, 84 strains of $B$. fragilis had Type IB, Type IIIB, or Type IIC CRISPR systems. Three strains (S38L3, S38L5, and S14) harbored all three types. In most bacteria, CRISPR systems are restricted to one or possibly two types but occasional strains harboring three types of CRISPR systems were seen in Streptococcus and Clostridium species (Louwen et al., 2014). In Streptococcus thermophiles, for example, three different major CRISPR-Cas systems are present and function independently in crRNA biogenesis (Carte et al., 2014).

Our final data set of $B$. fragilis CRISPR-Cas arrays, reflecting manual curation, is listed in Supplementary Table 1. The CRISPR repeat arrays are written with the oldest spacer at the top of the array; therefore the adjacent cas genes would be located proximal to the newest spacer, at the bottom of the array. The length and sequence of repeats and the length of spacers are generally well conserved within a CRISPR locus, but may vary between CRISPRs in the same or different genomes (Rath et al., 2015); CRISPR sequences may vary in both DR and spacer sequences even among strains more than $99 \%$ at the DNA level (Kunin et al., 2007). 
TABLE 2 | Distribution of CRISPR-Cas Systems in B. fragilis.

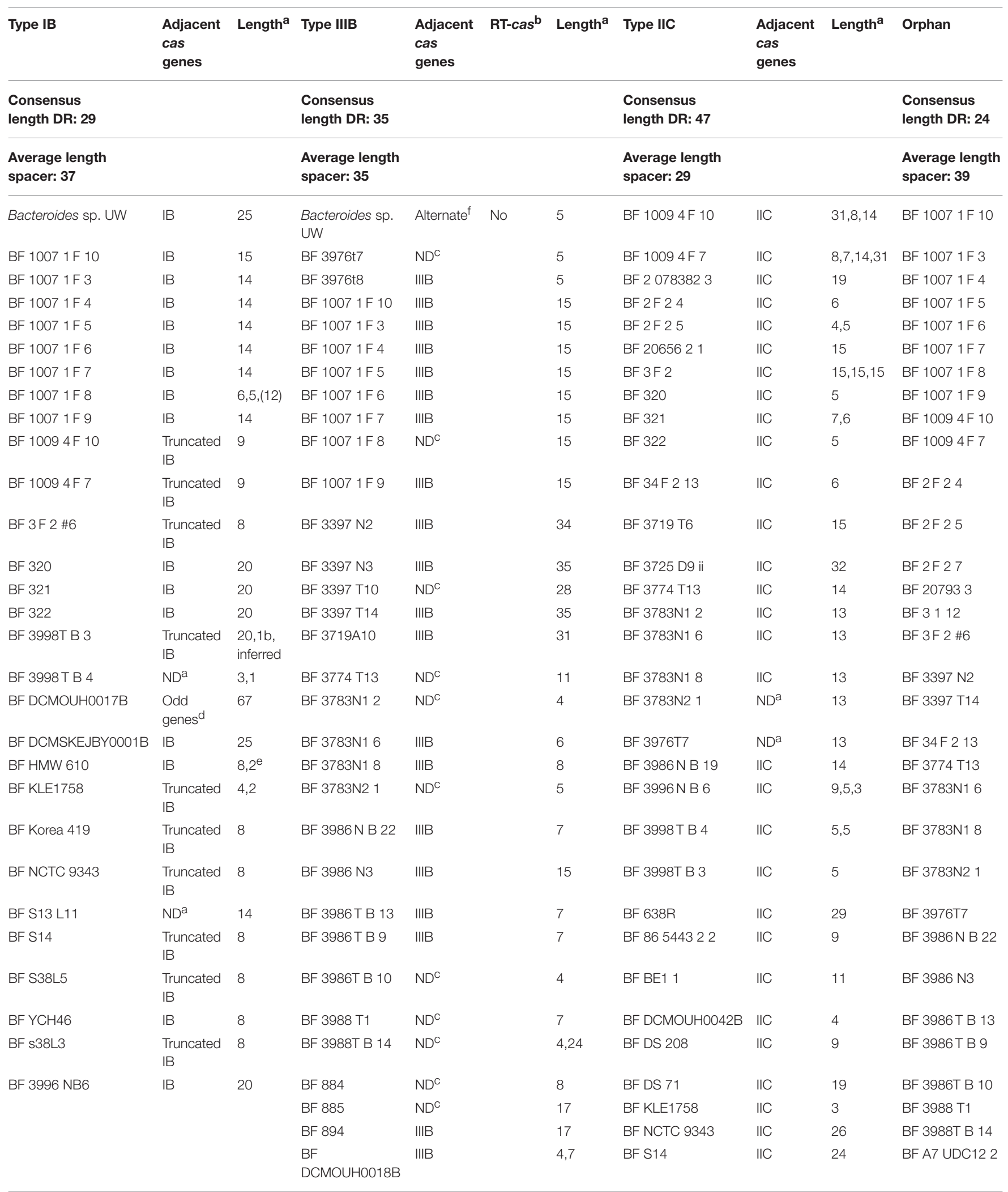


TABLE 2 | Continued

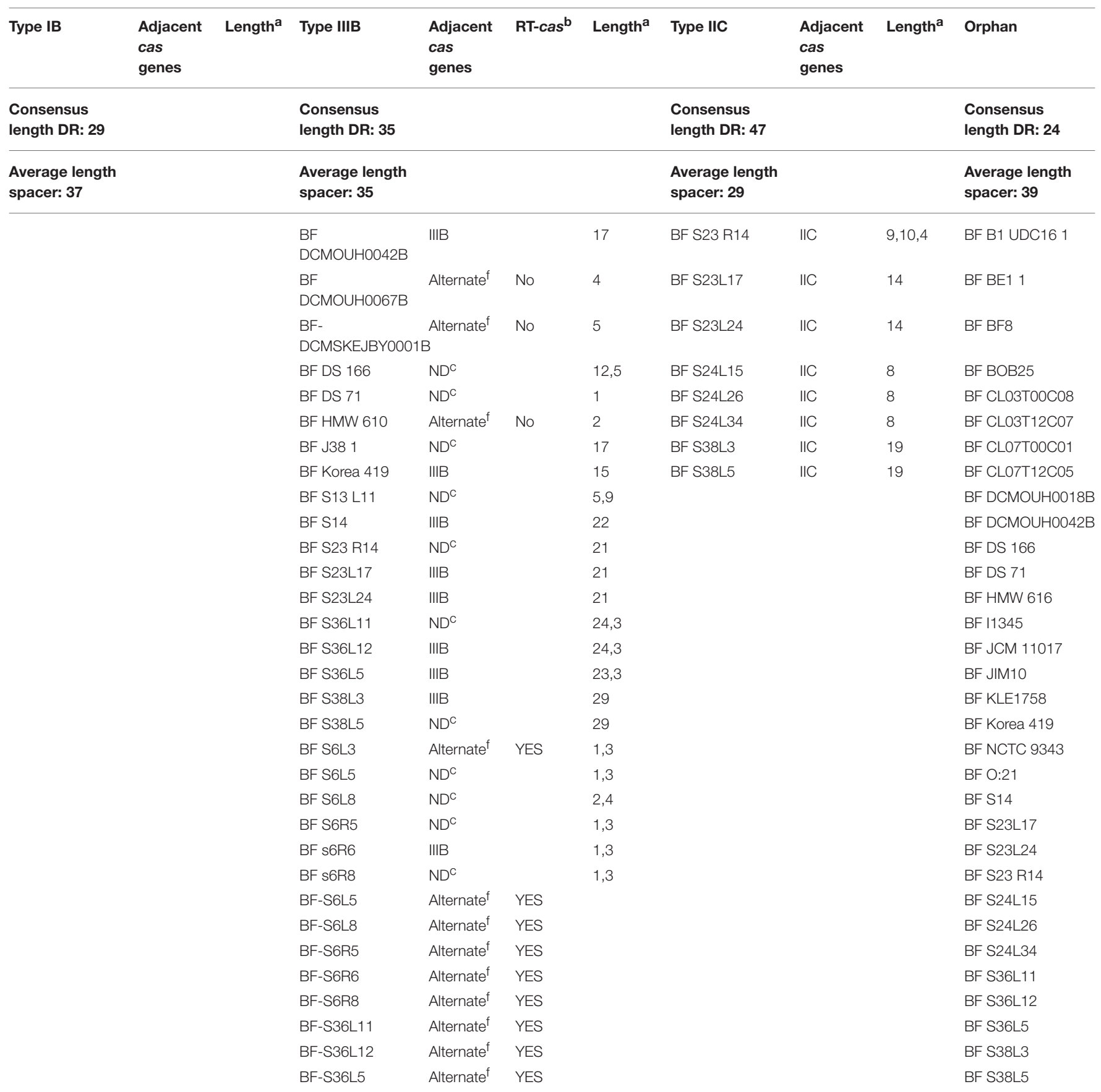

a Because many of the genomes are not yet assembled, the same CRISPR array may have been identified in different contigs, the length refers to the number of spacers in a particular CRISPR array.

${ }^{b}$ RT-cas: Gene coding for Reverse-transcriptase Cas1 fusion protein.

${ }^{c} N D$ : The contig on which the repeat array was found was very short and no adjacent cas genes could be identified.

${ }^{d}$ Has cas2, cas1, cas $4 a$ and cas 7 only; missing the effector cas genes.

${ }^{e} B F$ HMW 610 has large segments of N's in the midst of what appears to be a long, continuous repeat array.

$f$ Alternate gene neighborhood in the midst of polysaccharide and other metabolic genes.

\section{Class 1 Type IB CRISP-Cas Systems}

The closest match for the Type I Cas system is the canonic Type IB found in Clostridium kluyveri (Makarova et al., 2015;
Figure 2A) although the gene order is different. Using the traditional annotation servers, we found genes coding for Cas2, Cas1, Cas3, Cas4a, and Cas6 (TM1814). Three additional 


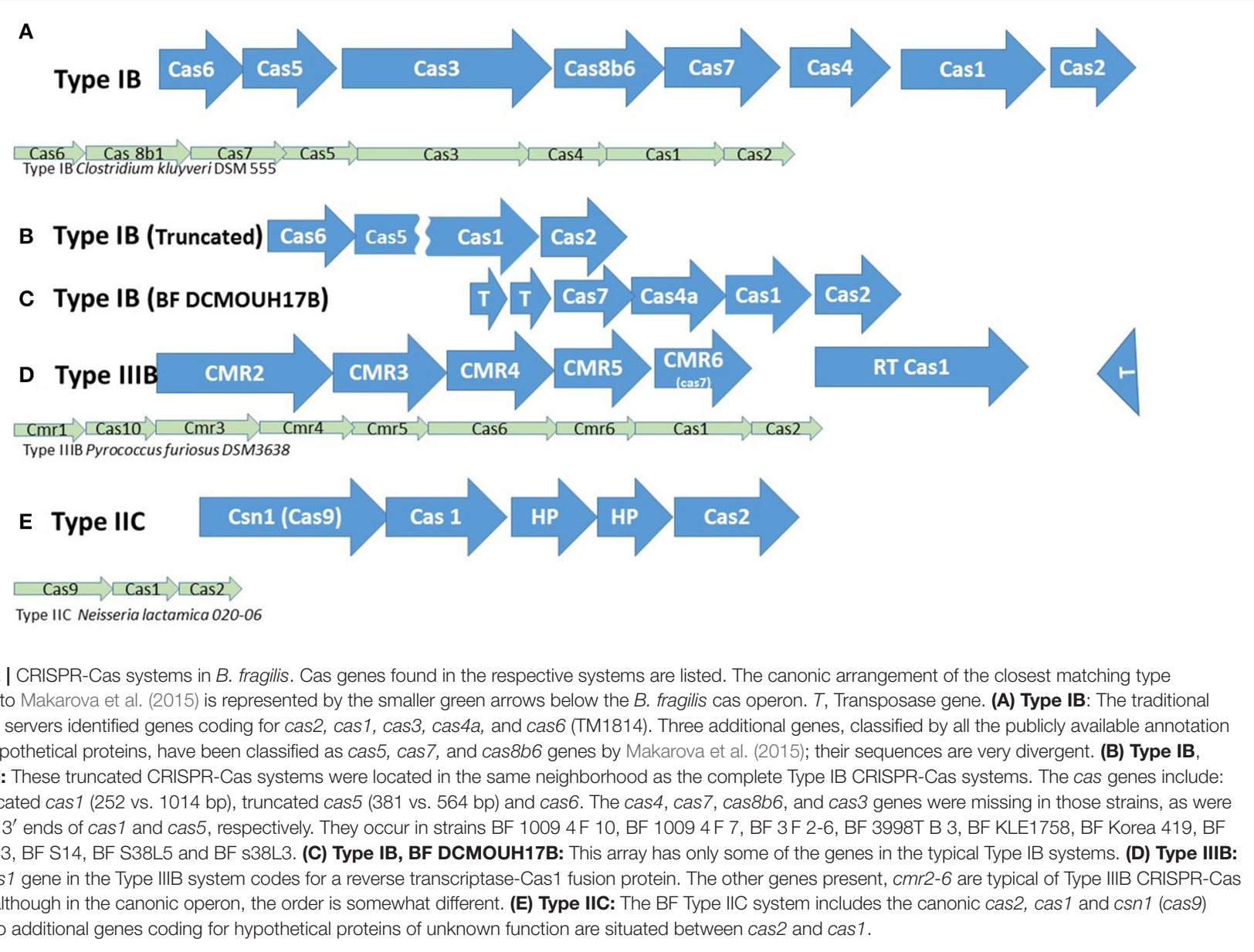

genes, classified by all the publicly available annotation sites as hypothetical proteins, were classified as cas5, cas 7 , and cas $8 b 6$ genes by Makarova et al. (2015) (Figure 2A); their sequences are very divergent (K. Makarova, personal communication). We did find annotated genes coding for Cas5 and Cas 8 in Bacteroides oleiciplenus YIT 12058, but their sequences did not have any homologs in B. fragilis isolates.

The Type IB cas 1 gene is highly conserved among $B$. fragilis strains. It is otherwise most closely aligned with cas 1 genes from other anaerobes, including Parabacteroides sp., Finegoldia magna, Clostridium tetani, and Clostridioides difficile) (data not shown). The Type IB cas 3 gene is also highly conserved among B. fragilis strains. Its closest matches were in other Prevotella, Porphyromonas, Chitinophaga, and Spirosoma species. It was highly divergent from other cas 3 genes in the model organism GenBank databank; its closest match was with Clostridiodes difficile (score 126, E-value: 1 e-28).

Twenty-nine strains of $B$. fragilis had Type IB CRISPRCas systems and were found in a highly conserved gene neighborhood (Figure 3A). These CRISPR-Cas systems could be divided into two main groups. In the first group, either a full set of Type IB cas operon genes could be identified upstream of the CRISPR repeat, or a portion of the operon was seen but was cut off because it was at the end of the sequenced contig; in those cases, the presence of a full TYPE IB cas operon was inferred. The second group had CRISPR repeats with 8 or fewer spacers and only a few cas genes (cas2, truncated cas1 (252 vs. $1014 \mathrm{bp}$ ), truncated cas5 (381 vs. $564 \mathrm{bp}$ ) and cas6) were adjacent to the CRISPR repeat. The cas4, cas7, cas $8 b 6$, and cas3 genes were missing in those strains, as were the $5^{\prime}$ and $3^{\prime}$ ends of cas 1 and cas5, respectively. The deletion of the cas genes, including cas 3 , and the truncation of the cas 1 and cas 5 probably occurred as a single deletion event, presumably after these spacers had been acquired, since the absence of the acquisition module probably crippled the ability of the strain to assimilate new spacer sequences. These truncated CRISPR-Cas systems, occurring in strains scattered across the dendrogram, were located in the same neighborhood as the complete Type IB CRISPR-Cas systems. All of the strains with the truncated CRISPR-Cas IB system have either Type IIIB or Type IIC systems, or both, so it is possible that the associated CRISPR-arrays could still use proteins encoded by those genes, despite their lack of the full set of Type IB cas genes.

Three blood isolates (Bacteroides sp. UW, BF DCMSKEJBY0001B and BF HMW610) had typical Type IB CRISPR-Cas systems (with adjacent cas genes including cas3) with relatively long repeat arrays (the array for HMW 610 was split into several segments because of N's in the sequencing results [data not shown]). The predicted Cas3 protein sequence 


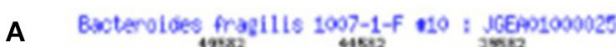

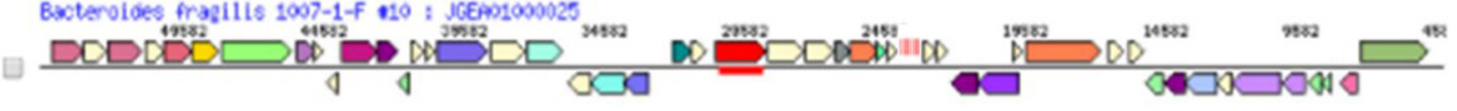

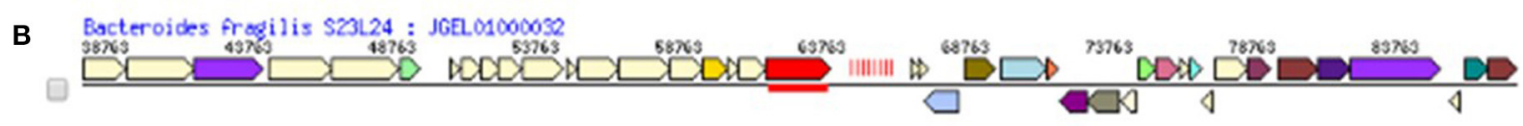

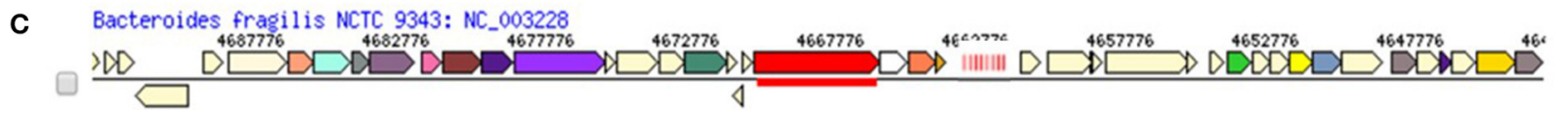

FIGURE 3 | Conserved gene neighborhoods of B. fragilis CRISPR-Cas arrays. Proteins coded for by genes are listed below. Vertical red lines represent repeat array. Associated cas genes products are in bold. Representative strain is shown with genes surrounding CRISPR-Cas array. The neighborhoods are highly conserved within each CRISPR-Cas type. The most characteristic cas gene for each group (i.e., A: cas 3, B: RT-cas 1-fusion and C: cas 9) are underlined.

(A) Conserved gene neighborhood of Type IB CRISPR-Cas system in B. fragilis. Upstream genes: Hypothetical protein;hypothetical protein;Manganese transport protein $\mathrm{MntH}$;Exodeoxyribonuclease III; hypothetical protein; hypothetical protein; hypothetical protein;Translation elongation factor LepA;putative Na+/H+ exchange protein; $\mathrm{Na}+/ \mathrm{H}+$ antiporter NhaA type;hypothetical protein;DNA recombination protein RmuC; Methionine aminopeptidase; CRISPR-associated protein, TM1814 family (cas 6); hypothetical protein (cas 5);CRISPR-associated helicase cas 3 (underlined); hypothetical protein; hypothetical protein;CRISPR-associated RecB family exonuclease cas 4a;CRISPR-associated protein cas 1;CRISPR-associated protein cas 2; CRISPR REPEAT ARRAY; Downstream genes: Hypothetical protein;hypothetical protein;RNA pseudouridylate synthase BT0642; RNA methyltransferase, TrmA family;hypothetical protein;Pyruvate,phosphate dikinase;hypothetical protein; hypothetical protein;Thiamin-phosphate pyrophosphorylase;Sulfur carrier protein adenylyltransferase ThiF;Thiazole biosynthesis protein ThiH; hypothetical protein;Thiamin biosynthesis protein ThiC; Thiazole biosynthesis protein ThiG;Thiamin-phosphate pyrophosphorylase; Sulfur carrier protein ThiS; Superoxide dismutase [Fe];ATP-dependent DNA helicase UvrD/PcrA;Carboxynorspermidine decarboxylase, putative; hypothetical protein;

(B) Conserved gene Neighborhood of Type IIIB CRISPR-Cas system in B. fragilis. Upstream genes: Two-component system sensor histidine kinase; Two-component system response regulator; Outer membrane protein assembly factor YaeT precursor; ABC transporter permease; Probable ABC transporter permease; putative $A B C$ transporter permease; putative $A B C$ transporter permease; $A B C$ transporter, permease protein; $A B C$ transporter, permease protein; $A B C$ transporter, permease protein; ABC transporter ATP-binding protein YvcR; Thiol:disulfide interchange protein; M. jannaschii predicted coding region MJ0978; protein of unknown function DUF88; hypothetical protein; hypothetical protein; hypothetical protein; hypothetical protein; CRISPR-associated RAMP Cmr2; CRISPR-associated RAMP Cmr3; CRISPR-associated RAMP Cmr4; CRISPR-associated RAMP Cmr5; CRISPR-associated RAMP Cmr6; Retron-type RNA-directed DNA polymerase RT-Cas1 fusion protein (underlined). CRISPR Repeat Array. Downstream genes: ISNCY family transposase; ISNCY family transposase; Aminotransferase class II, serine palmitoyltransferase like; Transcription regulator [contains diacylglycerol kinase catalytic domain]; Aspartyl-tRNA synthetase; hypothetical protein; $\mathrm{N}$-carbamoylputrescine amidase/Aliphatic amidase AmiE Agmatine deiminase;Ferredoxin domain containing protein; YbbL ABC transporter ATP-binding protein; YbbM seven transmembrane helix protein; hypothetical protein; Possible glyoxylase family protein (Lactoylglutathione lyase); hypothetical protein; Hypothetical protein Ybgl; Hypothetical protein; RND efflux system, outer membrane lipoprotein CmeC; RND efflux system, membrane fusion protein CmeA; RND efflux system, inner membrane transporter CmeB. In contrast, Gene Neighborhood of TYPE IIIB CRISPR Repeat Array in Blood Isolates (absence of cas genes) (not shown): Upstream Genes: Lipopolysaccharide biosynthesis protein; hypothetical protein;UDP-N-acetylglucosamine 2-epimerase; glycosyl transferase, group 1 family protein; hypothetical protein; mannosyltransferase B; UDP-N-acetylglucosamine 4,6-dehydratase; UDP-N-acetylglucosamine 2-epimerase; dTDP-4-dehydrorhamnose reductase; Glycosyl transferase, group 1 precursor; UDP-glucose 4-epimerase; Undecaprenyl-phosphate $\mathrm{N}$-acetylglucosaminyl 1-phosphate transferase; TYPE IIIB REPEAT ARRAY in BF BLOOD ISOLATES; Downstream genes: Putative non-specific DNA-binding protein; hypothetical protein; $\mathrm{Na}+/ \mathrm{H}+$-dicarboxylate symporter; 6-phosphogluconate dehydrogenase, decarboxylating; Glucose-6-phosphate 1-dehydrogenase; 6-phosphogluconolactonase, eukaryotic type; hypothetical protein;

(C) Gene Neighborhood of Type IIC CRISPR-Cas system in B. fragilis. Upstream gene neighborhood: Conserved hypothetical protein;putative transmembrane protein;putative transmembrane DNA mismatch repair-like protein;conserved hypothetical protein;putative urocanate hydratase;putative formimidoyltransferase-cyclodeaminase;putative imidazolonepropionase;putative formiminotransferase-cyclodeaminase;putative histidine ammonia-lyase;putative TetR transcriptional regulator; putative outer membrane efflux protein;putative membrane fusion protein transporter;putative transmembrane Acr-type transport protein;conserved hypothetical protein;putative transmembrane protein;conserved hypothetical protein;putative transmembrane polysaccharide modification protein; hypothetical protein;hypothetical protein;hypothetical protein; csn1(cas9) (underlined);conserved hypothetical protein (pseudogene);cas1;cas2; CRISPR REPEAT ARRAY. Downstream: Putative transmembrane protein;conserved hypothetical protein;putative transmembrane MotA/TolQ/ExbB proton channel family protein;conserved hypothetical protein;conserved hypothetical protein;putative TonB-family outer membrane receptor protein;conserved hypothetical protein;putative TPR-repeat family protein;putative ATP-binding component of ABC transporter; putative transmembrane protein;putative GntR family transcriptional regulator; conserved hypothetical protein;conserved hypothetical protein;putative ABC transporter transmembrane component.

of these three isolates cluster as a separate group in a phylogenetic analysis of Cas3 in B. fragilis as does Cas 6 and most of the other Cas proteins.

\section{Class 1 Type IIIB CRISPR-Cas Systems}

Fifty-six strains had Type IIIB CRISPR-Cas systems. The Type IIIB CRISPR-Cas system had a cas operon upstream of the repeat segment consisting of Cas1, CMR6, CMR5, CMR4, CMR3, and CMR2; a ISNCY (i.e., Not Classified Yet) transposase was frequently located at the opposite end of these CRISPRs (Figure 2B). The Cas1 gene in Type IIIB CRISPRs was often not annotated as Cas1 in either RAST or NCBI annotation but as a retron-type DNA polymerase or reverse transcriptase (RT). This cas1 gene (2271 bp) encodes a 756 aa protein that is more than 


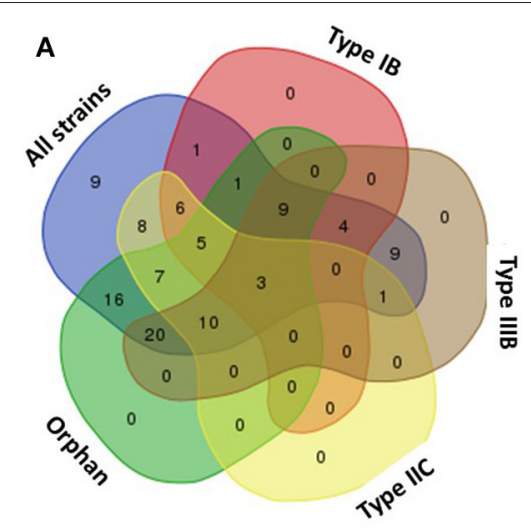

\section{B}

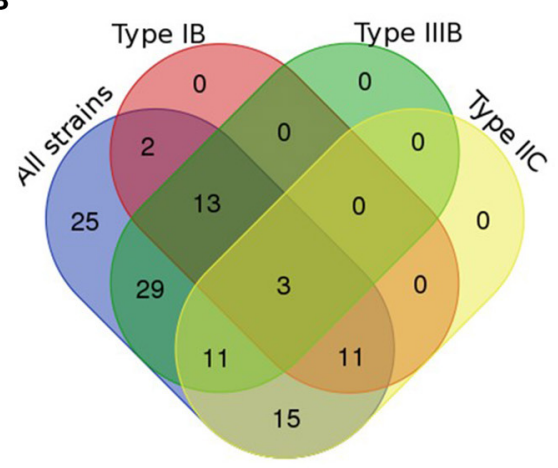

FIGURE 4 | Venn diagram of distribution of CRISPR-Cas systems among strains of $B$. fragilis. The Venn diagrams in Figure 4 include CRISPR-Cas repeats of a given type, whether full or truncated; whether or not there was a full set of adjacent cas genes is detailed in Table 2. (A) Distribution of all CRISPR arrays in B. fragilis. Orphan Type IB Type IIC Type IIIB-3 Strains: BF S38L3, BF S38L5, BF S14. Orphan Type IB Type IIIB-9 Strains: BF 1007 1 F 4 , BF 1007 1 F 3, BF Korea 419, BF 1007 1 F 6, BF 1007 1 F 7, BF 1007 1F 9, BF 1007 1 F 8, BF 1007 1F 10, BF 1007 1F 5. Orphan Type IB Type IIC-5 Strains: BF 1009 4F 7, BF KLE1758, BF 3-F-2 \#6, BF NCTC 9343, BF 1009 4F 10. Orphan Type IIC Type IIIB-10 Strains: BF 3783N1 8, BF 3783N2 1, BF 3976T7, BF DS 71, BF S23L17, BF S23L24, BF S23 R14, BF 3783N1 6, BF DCMOUH0042B, BF 3774 T13. Type IB Type IIIB-4 Strains: BF DCMSKEJBY0001B, BF S13 L11, BF HMW 610 Bacteroides sp. UW. Type IB Type IIC-6 Strains: BF 3996 N B 6, BF 321, BF 322, BF 3998T B 3, BF 320, BF 3998 T B 4. Orphan Type IB-1 Strain: BF YCH46. Type IIC Type IIIB-1 Strain: BF 3783N1 2. Orphan Type IIIB-20 Strains: BF 3986T B 10, BF DCMOUH0018B, BF S6L8, BF S36L12, BF 3986 T B 9, BF S6L3, BF DS 166, BF 3988T B 14, BF S6R8, BF S6L5, BF S36L5, BF 3986 N B 22, BF 3988 T1, BF S6R6, BF 3397 N2, BF S36L11, BF 3397 T14, BF 3986 T B 13, BF S6R5, BF 3986 N3. Orphan Type IIC-7 Strains: BF 34 F 2 13, BF 2 F 2 4, BF 2 F 2 5, BF S24L26, BF S24L34, BF S24L15, BF BE1 1. Type IB-1 Strains: BF DCMOUH0017B. Type IIIB-9 Strains: BF 3397 T10, BF 3976 T8, BF DCMOUH0067B, BF J38 1, BF 894, BF 885, BF 884, BF 3719A10, BF 3397 N3. Type IIC-8 Strains: BF 3719 T6, BF 3725 D9 ii, BF 2078382 3, BF 206562 1, BF DS 208, BF 3986 N B 19, BF 638R, BF 8654432 2. Orphan-16 Strains: BF BOB25, BF A7 UDC12 2, BF CL07T00C01, BF CL03T00C08, BF HMW 616, BF 2 F 2 7, BF 31 12, BF CL07T12C05, BF CL03T12C07, BF JCM 11017, BF JIM10, BF, BF8, BF I1345, BF 20793 3, BF B1 UDC16 1, BF O: 21. No CRISPR-Cas- 9 Strains: BF CL05T00C42, BF HMW 615, BF DCMOUH0085B, BF Ds 233, BF 3725 D9, BF CL05T12C13, BF J 143 4, BF 4g8B, BF 2d2A. (B) Distribution of CRISPR-Cas systems excluding orphan CRISPR arrays. Type IB Type IIC Type IIIB-3 Strains: BF S38L3, BF S38L5, BF S14. Type IB Type IIIB-13 Strains: BF 1007 1F 4, BF DCMSKEJBY0001B, BF 1007 1F 3, BF Korea 419, BF S13 L11, BF 1007 1F 6, BF 1007 1F 7, BF HMW 610 Bacteroides sp. UW, BF 1007 1 F 9, BF 1007 1 F 8, BF 1007 1F 10, BF 1007 1 F 5. Type IB Type IIC-11 Strains: BF 3996 N B 6 , BF 321 , BF 1009 4F 7 , BF KLE1758, BF 3-F-2 \#6, BF 322, BF 3998T B 3, BF 320, BF NCTC 9343, BF 10094 F 10, BF 3998 B 4. Type IIC Type IIIB-11 Strains: BF 3783N1 8, BF 3783N2 1, BF 3976T7, BF DS 71, BF S23L17, BF 3783N1 2, BF S23L24, BF S23 R14, BF 3783N1 6, BF DCMOUH0042B, BF 3774 T13. Type IB-2 Strains: BF YCH46, BF DCMOUH0017B. Type IIIB-29 Strains: BF 3397 T10, BF 3986T B 10, BF DCMOUH0018B, BF 3976 T8, BF DCMOUH0067B, BF S6L8, BF J38 1, BF S36L12, BF 3986 T B 9, BF S6L3, BF DS 166, BF 3988T B 14, BF 894, BF S6R8, BF S6L5, BF S36L5, BF 3986 N B 22, BF 885, BF 3988 T1, BF 884, BF S6R6, BF 3397 N2, BF 3719A10, BF S36L11, BF 3397 N3, BF 3397 T14, BF 3986 T B 13, BF S6R5, BF 3986 N3. Type IIC-15 Strains: BF 34 F 2 13, BF 3719 T6, BF 3725 D9 ii, BF 2 078382 3, BF 206562 1, BF DS 208, BF 3986 N B 19, BF 2 F 2 4, BF 638R, BF 2 F 2 5, BF S24L26, BF 86 5443 2 2, BF S24L34, BF S24L15, BF BE1 1. No CRISPR-Cas (with adjacent cas genes)-25 Strains: BF CL05T00C42, BF BOB25, BF A7 UDC12 2, BF CL07TO0C01, BF CL03T00C08, BF HMW 615, BF HMW 616, BF 2 F 2 7, BF 31 12, BF DCMOUH0085B, BF CL07T12C05, BF CL03T12C07, BF JCM 11017, BF Ds 233, BF JIM10, BF, BF8, BF 3725 D9, BF CL05T12C13, BF J 143 4, BF 11345, BF 4g8B, BF 2d2A, BF 20793 3, BF B1 UDC16 1, BF O: 21.

twice as long as the 243 aa Cas 1 protein encoded by the cas 1 gene in the Class 1 Type IB CRISPRs. These proteins contain both an RT-like superfamily domain and a Cas1_I-II-II superfamily domain (the amino acid sequence of RT-like Cas1, when present, is almost completely conserved among $B$. fragilis strains).

The RT-Cas protein is highly conserved within strains of B. fragilis, but very divergent from other RT-Cas 1 proteins. When compared to the landmark organisms (proteomes from 27 genomes spanning a wide taxonomic range) at NCBI, the closest matches were Clostridiodes difficile 630, fission yeast and Sulfolobus acidocaldirius (data not shown). If a wider search of the NR database was done and Bacteroides species eliminated, the closest matches were Culturomica, Candidatus, Sulfuromonas, and Porphyromonas species.

The $B$. fragilis Type IIIB CRISPR-Cas systems occur in highly conserved gene neighborhoods in the strains in which they are found (Figure 3B); these neighborhoods include a high percentage of genes involved in efflux processes. Frequently, a transposase gene was located just downstream of the CRISPR repeat region in those strains with adjacent Type IIIB CRISPRCas operons. In some cases, the contig ended just downstream of the RT-caslgene; this type of sequencing result is frequently due to the presence of a transposase gene that causes breaks in the automatic scaffolding assembly of genome sequences. Transposase genes are often part of MGEs but other genes typical of mobile elements were not seen in these genomic neighborhoods. It is conceivable that the transposase gene has some function in the formation of the adjacent CRISPR (including transfer via HGT) but we could not find any mention of an association of this kind in the literature to date. The implications of the proximity of the CRISPR to the particular genomic neighborhood, if any, are unknown.

Some of the strains had Type IIIB CRISPR repeat arrays without adjacent cas genes in a different gene neighborhood; these included B. fragilis blood isolates as well as several ETBF isolates (in the ETBF isolates there were two type IIIB CRISPR arrays, one in the conserved neighborhood with adjacent cas genes and one in the same alternate neighborhood as the arrays 
in the $B$. fragilis blood isolates). These arrangements are discussed further below (virulence association with CRISPR-Cas types).

\section{The Type IIIB B. fragilis CRISPR/Cas System with the Cas1-RT Fusion Protein Are Not Common among Bacteria}

A GenBank search for proteins with both RT and Cas1 domains revealed that only $8 \%$ of bacterial Type IIIB elements have a Cas $1 /$ reverse transcriptase fusion protein, and these were most prevalent among cyanobacteria (Silas et al., 2016). The limited phylogenetic distribution of Cas1-RT and its association with only one CRISPR type suggests that there are a small number of origins of these RT-Cas1 fusions (Makarova et al., 2006; Silas et al., 2016). Recent data supports the notion that this protein may provide an efficient method to facilitate acquisition of spacers directly from RNA (Silas et al., 2016). Since other Type III CRISPR systems target RNA for degradation, these RT-associated CRISPR-Cas systems would effectively generate adaptive immunity against RNA parasites.

Further, this mechanism could target highly transcribed regions at both the DNA and RNA levels and thus serve in a regulatory capacity. The ability to target RNA substrates without targeting the bacterial chromosome would be a useful method for CRISPR regulation of endogenous genes (Sampson and Weiss, 2014). In B. fragilis, the conserved gene neighborhood of the Type IIIB CRISPRs included efflux operons, genes involved in cell wall biosynthesis and division, and/or genes involved in iron transport and storage.

\section{Class 2 Type IIC CRISPR-Cas Systems}

Forty strains had Type IIC systems that contained the cas2, cas1, and $\operatorname{csn} 1$ (cas9) genes (Figure 2C). For most of the strains, there are additional ORFs between cas 1 and cas 9 that encode two hypothetical proteins annotated as putative DNA binding proteins in a cluster with Type 1 RestrictionModification systems. These hypothetical proteins have a conserved domain annotated as RhuM; this is a group of proteins implicated in virulence/pathogenicity (RhuM, of unknown function, is encoded in the SPI-3 pathogenicity island in Salmonella typhimurium). Fourteen percent $(46 / 320)$ of the spacers could be identified in phage sequences (Supplementary Table 2C).

Csn1 (Cas9) proteins in the Type IIC systems were highly conserved among strains of B. fragilis ( $>99 \%$ identity). The closest homologs (52-70\% identity) were found in other species including Parabacteroides, other Bacteroidetes, Coprobacter, Capnocytophaga, and Prevotella species. Csn1 (Cas9) belongs to the COG3513 - CRISPR/Cas system Type II associated protein, and contains $\mathrm{McrA} / \mathrm{HNH}$ and RuvC-like nuclease domains.

Many of the Type IIC CRISPR-Cas arrays were found on contigs that ended just downstream of the CRISPR repeat, so not much information could be gleaned about the downstream neighborhood. The upstream gene neighborhood, when it could be discerned, was highly conserved (Figure 3C).

\section{Short CRISPR Repeats with 3 DRs and No Adjacent cas Genes (i.e., Orphan CRISPRs) Were Found Upstream of the hipB Gene in 71 Strains of $B$. fragilis}

The entire sequence was completely conserved in these isolates, which originated from a variety of different sources, including normal feces, human microbiome project isolates, ETBF isolates, blood and virulent/MDR isolates. The hipAB operon is thought to be important in development of persister cells and multidrug tolerance in chronic infections due to $E$. coli and other bacteria (Day et al., 2004; Correia et al., 2006; Schumacher et al., 2015). HipA inhibits protein synthesis resulting in inhibition of cell growth and leading to multidrug tolerance by driving the cells into reversible dormancy and resulting in the production of persister cells (Schumacher et al., 2015). HipB binds HipA and acts as a transcriptional repressor of the hipBA operon (Hansen et al., 2012) and regulation of this operon is a key factor in controlling persister formation. Recently, high persister mutations were found in E. coli in which the mutation was not in the active site but rather interfered with higher order HipAHipB promoter complexes that occluded the active site, thereby "unleashing" HipA to effect multidrug tolerance (Schumacher et al., 2015).

Tight regulation of hipAB is important since dormancy is only desirable if bacterial viability is threatened. Indeed, in global transcriptome studies of several $B$. fragilis strains we found that the hipA homologs were transcribed but at a very low level (manuscript submitted for publication). The ubiquitous proximity of this short orphan CRISPR to the hipAB operon in $B$. fragilis and the lack of adjacent cas genes raises the possibility that this "orphan" CRISPR is involved in regulation of hipAB but experimental analysis is necessary to determine whether this is the case. If confirmed, this would constitute a novel mode of regulation and could be important in the recalcitrance of chronic $B$. fragilis infections to antibiotic treatment. We did not find any significant repeat region upstream of hip $A B$ in bacteria with genes phylogenetically related to $B$. fragilis hipA (E. coli NEB5A_07695, E. coli 0104: H4 str. 2011c-3493, Shewanella oneidensis MR1, Streptomyces coelicolor and Myxococcus xanthus). Orphan CRISPRs (i.e., without associated cas genes) have been implicated in regulation in other bacteria (Sampson and Weiss, 2014). In some cases, the spacers within the CRISPR target endogenous genes and there are no associated cas genes. Whether the lack of adjacent cas genes explains why the chromosome itself is not targeted (Stern et al., 2010), or whether these CRISPRs may use CAS proteins from other locations and still be involved in regulation of the targeted genes or gene transcripts remains unclear.

\section{Different CRISPR/Cas Subtypes Have Associated DR Sequence Subtypes and May Be Associated with Specific Functions in the Cell}

This agrees with patterns seen in other bacteria (Kunin et al., 2007; Makarova et al., 2015) and is consistent with the notion 
that different sets of genes are needed to recognize, bind and process the different repeat types; the differences are probably related to the particular fold structure assumed by the DR sequence. Consensus sequences of the repeat sequence for the four CRISPR-Cas systems, their placement within the database of CRISPR direct repeat sequences, and their predicted associated RNA structure are shown in Figures 5 and 6 . Figure 5 shows the placement of the four consensus repeat sequences within the entire Direct Repeat database as well as the nearest phylogenetic neighboring DRs. Notably, the four CRISPR consensus DRs were located across the entire DR spectrum, with phylogenetic neighbors from very distantly related species. More details about the phylogenetic placement of the DRs can be found in the legend to Figure 5.

The length of the consensus repeat sequence differed among the CRISPR-Cas types; Type IB (29 bp), Type IIIB (35 bp), Type IIC (47 bp), and Orphan (24bp). This is in agreement with the finding that unusually long repeats (up to $48 \mathrm{bp}$ ) are exclusively present in Type IIC systems, especially in the Bacteroidetes phylum (Chylinski et al., 2014); the average CRISPR repeat in this system among all prokaryotes is 36 bp (Chylinski et al., 2013). The predicted fold structures of the DR sequences in each of the different CRISPR-Cas types was distinctive (Figure 6).

\section{Identification of Protospacers and Spacer Distribution within Each of the CRISPR Types Are Shown in Table 2}

The search itself is somewhat problematic because the spacer sequences are so short. We used both the CRISPR Target program and NCBI BLAST with specified parameters to search for protospacer matches. Occasionally, a particular gene was identified to which the spacer had significant homology but, more often, other CRISPR arrays were identified. We repeated the search using the NCBI BLAST engine but adding a filter to screen out Repeat Arrays; we were able to identify protospacer matches for some additional spacer sequences but more often the match was to non-related nucleotide sequences.

We found that $7 \%$ of the spacer sequences were significantly homologous to a Bacteroides phage sequence. The majority of protospacers could not be assigned to a specific sequence (phage, plasmid or other bacterial gene or even inter-gene sequences) and most were identified in CRISPR regions in other B. fragilis strains. These numbers are consistent with other studies of prokaryotic CRISPR-Cas arrays; for example, in Riemerella strains, only $13 / 153(8 \%)$ of spacers were homologous with a phage or plasmid target (Zhu et al., 2016).

In closely related strains, spacer identity and placement was frequently highly conserved. Spacers common to multiple strains can be due to either clonal dispersion of the CRISPR element through heredity or to HGT of these CRISPRs. The identified spacers, along with the strains in which they are found, their placement in those strains, and their predicted protospacer target are listed in Supplementary Tables 2A-C. A visual representation of their distribution is shown in Supplementary Tables 3A-C (binary distribution tables in Excel format).
One hundred thirty three unique spacers were found in the Type IB CRISPR-Cas systems. Five of the spacer targets matched, imperfectly but across the entire spacer length, to a phage sequence; these spacers were all found in an atypical, extremely long (67 spacers!) CRISPR repeat array in the blood isolate BF DCMOUH0017B. Notably, this array had an incomplete set of adjacent cas genes (only cas1, cas2, and cas4) and two transposases (one IS21 type) immediately upstream of cas1. Cas1, Cas2, and often Cas4 comprise the adaptation module that is responsible for new spacer insertion (Makarova et al., 2015). The very long length of this CRISPR repeat array and multiple spacers matching $B$. fragilis phage protospacers is consistent with a CRISPR-Cas system that can acquire new spacers but cannot defend against the invading nucleic acid.

Type IIC systems contained significantly higher portion of spacers with crRNA homologous to a phage sequence than were found in the other systems ( $87 \%$ of spacers matching a phage sequence belonged to Type IIC CRISPR-Cas systems, 13\% belonged to Type IB systems).

Spacer distribution in the Type IIIB CRISPR-Cas systems is shown in Supplementary Table $2 \mathrm{~B}$ and graphically in Supplementary Table 3B. There were 252 unique spacers in the Type IIIB system, and the distribution was very broad. Strains with close phylogenetic relationship had identical spacers (e.g., BF 1007 F-2 thru F-10; BF 320, BF 321, and BF 322). Fifteen of the spacers $(15 / 252,6 \%)$ had matches with prokaryotic genes; no matches with phage or plasmid genes were identified and most matches were with other CRISPR regions (Supplementary Table 2B).

Type III CRISPR-Cas systems are associated with degradation of phage DNA in Staphylococcus epidermidis (Jiang et al., 2016). However, in Streptococcus pyogenes, there is a strong bias of the Type II system for acquisition of spacers matching viral protospacers (Heler et al., 2015). In our study, as in others, the DR repeat structures and associated cas genes of the two types were very distinct. Apparently, more than one CRISPR-Cas systems with the associated DR repeat structure can be used for acquisition and/or degradation of phage genetic material.

\section{Leader Sequences in CRISPR-Cas Systems}

A 193 bp leader sequence was identified in the Type IB system of BF YCH 46 using CRISPRleader version 3.0 (Alkhnbashi et al., 2016) (kindly analyzed for us by Dr. Omer Alkhanbashi). The sequence was located between the repeat array and the cas genes and was extremely conserved among the Type IB CRISPR-Cas systems identified in B. fragilis. (Leader sequence: (5'-TGTTATTGTGAATTATCAATGGTAAAGTAACGGAAAC GCTCTATGACATGTTGATATATAGATGTTTAGTACCTATG TCGCTAACCTATGTTTTTTATATTATTCTTGATCGACACA TTATTTCTAAAGAAAAGCAATTTTCTGCAAAAGCATTTG GCTTATTACTAAGTAATTGCGTTGATTGATGGGTAGA-3'). Strains BF HMW610, Bacteroides sp. UW, BF DCMSKEJBY0001B and BF DCMOUH0017B, all phylogenetically related BF blood isolates, had slightly divergent Type IB leader sequences (data not shown). The promoter sequences for $B$. fragilis are not completely established and no 


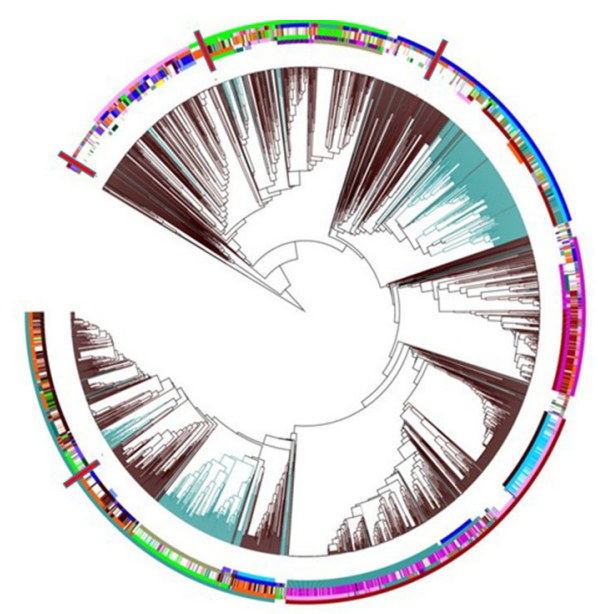

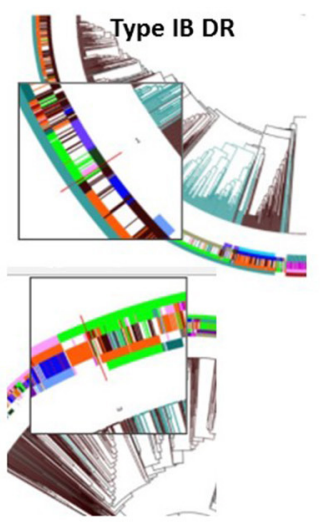

Type IIC DR
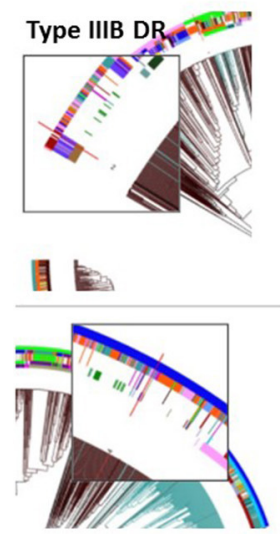

Orphan DR

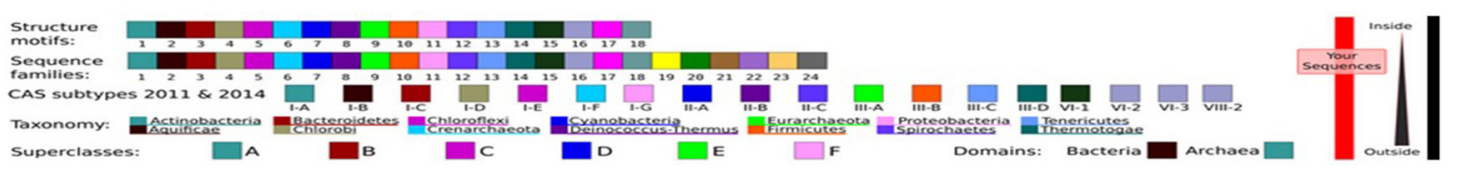

FIGURE 5 | Relationship of $B$. fragilis DR (direct repeat) sequence to other sequences in the Direct Repeat Database. CRISPR Map was used to locate the consensus sequences of the four CRISPR-Cas types of B. fragilis. Based on placement within the map shown, Superclass, tentative taxonomy, Cas subtype and Sequence Family were determined (if available). Based on a detailed tree (not shown) of all of the DRs in the database, the closest phylogenetic neighbors were determined. BF1_IB: Superclass: A, Taxonomy: Bacteroidetes, Cas subtype: IB, Sequence Family: 2 (158 bacteria including 2 strains of BF). Nearest phylogenetic neighbors: Phormidium sp. (cyanobacteria living at temperatures of 45-60) and Pyrococcus yayanosii (strictly anaerobic, hypertermophilic archeon isolated from the deep sea); BF2_IIC: Superclass: -, Taxonomy: Bacteroidetes, Cas subtype IIC, Sequence family: 21 (23 bacteria including 2 DRs of B. fragilis strains). Nearest phylogenetic neighbors: Capnocytophaga, a gram-negative bacterium (Phylum Bacteroidetes, Family Flavobacteriaceae) normally found in the oropharangeal tract of mammals and involved in pathogenesis of animal bite wounds and periodontal disease. Remarkably, Capnocytophaga carries cfxA and cepA, two $\beta$-lactamase genes found in Bacteroides species and responsible for $\beta$-lactam resistance in Bacteroides. Phylogenetic analysis indicated that the Cas 9 protein was also closely related to that of Cas9s found in Capnocytophaga; another close match was to Fluviicola taffensis, a novel freshwater bacterium of the family Cryomorphaceae within the phylum "Bacteroidetes"; BF3_IIIB: Superclass: E; Taxonomy: Firmicutes, Cas subtype IIIA? (based on arrangement of the cas genes, we assigned this DR to CRISPR-Cas Type IIIB). Nearest phylogenetic neighbors: Saprospira grandis, a gram-negative, marine, multicellular, filamentous flexibacterium, (phylum Bacteroidetes, Class: Sphingobacteria) known for devouring bacteria (and algae) and Methanococcus vaniellii (Superkingdom Archea, Phylum Euryarchaeota); both (particularly the latter) indicates that the CRISPR may have been horizontally transferred from a phylogenetically distant species; BF4_Orphan Superclass: D Taxonomy: Proteobacteria. Nearest phylogenetic neighbors: Fluviicola taffensis, a novel freshwater bacterium (Phylum Bacteroidetes, family Cryomorphaceae) and Ornithobacterium rhinotracheale (Phylum Bacteroidetes, family Flavobacteriaceae) a bacterium found worldwide that causes potentially fatal respiratory disease in poultry.

obvious promoter sequence was found on the leader sequence (Bayley et al., 2000). While no specific leader sequence could be definitively assigned in the Type IIIB or Type IIC systems using the CRISPRleader program, the sequences upstream of the CRISPR repeat array (and downstream of the cas operon) were highly conserved, respectively, in each of those two CRISPR-Cas types. As was found for the Type IB leader, strains BF HMW610, Bacteroides sp. UW, BF DCMSKEJBY0001B and BF DCMOUH0017B had slightly divergent leader sequences for both Types IIIB and IIC systems.

\section{No "Typical" CRISPR Systems Could Be Identified on Mobile Elements}

Phylogenetic analysis indicates that CRISPR-Cas arrays have undergone extensive HGT, possibly on megaplasmids, as very similar cas genes are found in distantly related organisms (Godde and Bickerton, 2006). CRISPR-Cas arrays were found on a variety of MGEs (Sorek et al., 2008) including Clostridium butyricum megaplasmids (Iacobino et al., 2013) and viruses attacking Cyanobacter (Chenard et al., 2016). We examined a variety of mobile elements in $B$. fragilis for the presence of CRISPR arrays. Ten plasmids and/or conjugative transposons for which there is partial or complete sequence were examined by CRT and did not have any discernable CRISPRs. These included: pBF9343, CTnHybL, pHAG88, p610 88, BOB_25 PAO, CTn86, CTn341, CTnDot, and CTnPg1-a (note: many of the conjugative transposons in $B$. fragilis were only partially sequenced). Additionally, no CRISPRs were detected in the large horizontally transferred chromosomal fragments recently described (Husain et al., 2017). We also examined available sequences for $14 \mathrm{~B}$. fragilis plasmids and did not find any CRISPR elements contained within their sequences. The examined plasmids (and their Genbank accession numbers) were: 2-078382-3, pBFP53, complete sequence, (CM004523.1); DCMOUH0042B, pBFU42e contig 1, (JPGQ01000001.1); pBFU42e, insertion sequence ISBf13 (complete), (KJ417513.1); pBF69566b, (KJ830768.1); pBF69566a, (KJ830769.1); 20656-2-1, pBI143, (LIDU01000064.1); IS4351, $\mathrm{R}$ plasmid encoding 


\section{A Type IB}
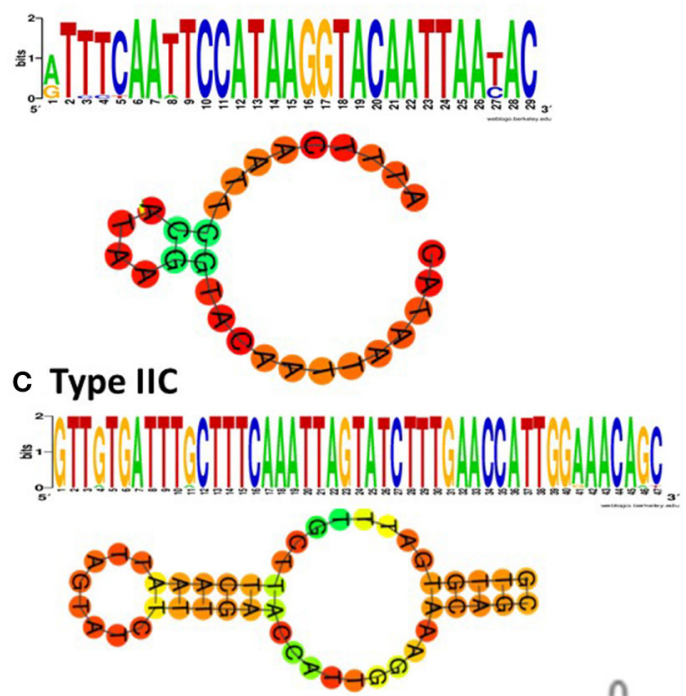

B Type IIIB

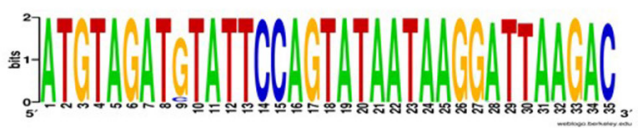

D Orphan

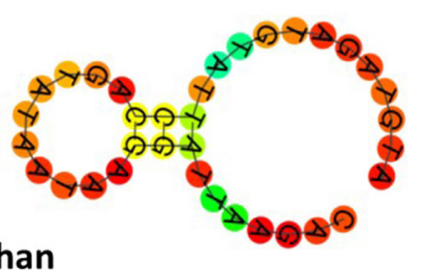

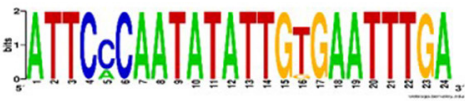

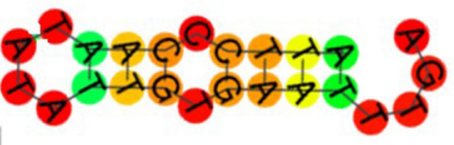

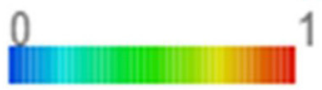

FIGURE 6 | Consensus direct repeat sequences and predicted fold structure for CRISPR-Cas systems in B. fragilis. The structure is colored by base-pairing probabilities. For unpaired regions the color denotes the probability of being unpaired. A short bar denoting the base pairing probability is including in the drawing. (A) Type IB Direct Repeat. The centroid secondary structure in dot-bracket notation has a minimum free energy of $0.10 \mathrm{kcal} / \mathrm{mol}$. (B) Type IIIB Direct Repeat. The centroid secondary structure in dot-bracket notation has a minimum free energy of $0.10 \mathrm{kcal} / \mathrm{mol}$. (C) Type IIC Direct Repeat. The centroid secondary structure in dot-bracket notation has a very stable secondary structure with a minimum free energy of $-5.30 \mathrm{kcal} / \mathrm{mol}$. (D) Orphan Direct Repeat. The centroid secondary structure in dot-bracket notation has a minimum free energy of $-0.90 \mathrm{kcal} / \mathrm{mol}$.

macrolide B resistance, (M17124.1); JIM 10, unnamed plasmid, (MBRB01000037.1); JIM 10, unnamed plasmid, (NZ_CM004507.1) pBFP35, complete sequence, (NC_011073.1); pBFUK1, complete sequence, (NC_019534.1);; 2-0783823, pBFP53, (NZ_CM004523.1); DCMOUH0042B,pBFU42e contig 1, (NZ_JPGQ01000001.1); pBI143, complete sequence, (U30316.1). No CRISPRs could be identified on the two sequenced BF phages (B40-8 and B124-14). However, using the more relaxed parameters in the CRISPR finder program, a potential CRISPR repeat was identified in PBF9343 but was not associated with adjacent cas genes. It should be noted that many of the CRISPRs that were identified on the genome had adjacent genes (e.g., transposase, mobility ( $m o b$ ) genes) that are typically found in mobile elements, so it is certainly possible that these CRISPRs are MGE-associated but that the exact MGE is not defined.

\section{Transposase Genes Were Frequently Found Adjacent to CRISPR Systems}

Since the CAS proteins themselves possess transposase activity, it is not clear why it would be advantageous to have another transposase gene in close proximity. The presence of these genes, which are ubiquitous on mobile elements, might suggest that these CRISPRs are (or were in the past) contained within a putative MGE. There are ancestral innate immune systems that were formed from transposon-like elements containing cas 1 and cas2, eventually using the terminal inverted repeats characteristic of the transposon to form the ancestral CRISPR repeats that were then duplicated by cas1 (cas1 also functioned in addition of spacers) (Nuñez et al., 2015). The molecular mechanism of CRISPR spacer integration is similar to that of both retroviral integration and DNA transposition that are mediated by integrases/transposases (Rath et al., 2015). Thus, it is possible that this adjacent transposase has some sort of function with the CRISPR function itself; however, that is purely speculative.

\section{Association of Virulence in $B$. fragilis Strains with Specific CRISPR-Cas Systems}

The presence of CRISPR-Cas systems are variably associated with virulence (Makarova et al., 2011; Barrangou, 2015) and/or antibiotic resistance in pathogenic bacteria. On the one hand, the ability of a bacterium to incorporate mobile elements bearing pathogenic determinants would argue for a less robust CRISPR-Cas defense system; on the other hand, the presence of multiple CRISPRs systems with a variety of spacers may indicate previous exposure to these elements, parts of which were incorporated into the bacterial genome.

The B. fragilis strains isolated from blood (Table 1) that clustered into a tight phylogenetic group (Figure 1) had distinctive properties in their CRISPR-Cas content. None of these strains, with the exception of BF DCMOUH0042B (which clearly has a different lineage) had CRISPR-Cas systems belonging to the 
Type IIC CRISPR-Cas system. The distinct lack of the Type IIC CRISPR-Cas system for the majority of the blood isolates might suggest that the ability to incorporate phage DNA (which often carries antimicrobial resistance or virulence genes) is a beneficial trait for these virulent strains.

Further, the while these isolates did contain Type IIIB CRISPR repeat arrays, they differed from the typical Type IIIB array in two ways: (1) they were located in a completely different gene neighborhood (Figure 3B) and (2) had no adjacent cas genes. Interestingly, several of the ETBF isolates contained two sets of Type IIIB CRISPR-Cas repeat arrays: one in the typical Type IIIB gene neighborhood with a full set of adjacent Type IIIB cas genes and one (without adjacent cas genes) in the same neighborhood as the Type IIIB blood isolate CRISPR arrays.

No significant target sequences for the blood isolate spacers were identified, except for one spacer in BF DCMOUH0018B with significant homology to a region carrying the $B$. fragilis insertion sequence IS1168 and $\operatorname{nimB}$ (nitroimidazole resistance) gene. The particular spacers carried by these isolates, despite the lack of Type IIIB cas genes, could be due either to them acquiring them at some point via an intact Type IIIB CRISPR-Cas system that they subsequently lost (perhaps during the transfer to an alternate gene neighborhood) or by HGT of the repeat array (without the cas genes) from another strain that did contain those spacers.

Figure 7A is an excerpted panel of the full binary representation of spacer distribution in Supplementary Table 3B and highlights the spacer distribution of the atypical Type IIIB CRISPR-Cas arrays in blood isolates (red font) as well as in those isolates that have both typical and atypical Type IIIB systems. Each unique spacer is represented by a red vertical bar. Four of the blood isolates have no other Type IIIB CRISPR-Cas array and no Type IIIB cas genes anywhere on the genome. Some of the ETBF isolates also have an array containing these same spacers, and also in the same "atypical" neighborhood. But these isolates also have another Type IIIB array, in the traditional
A strain

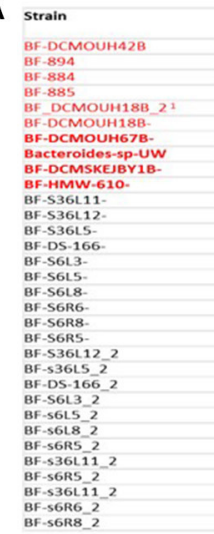

Cas IIB
genes
YES
YES
YES
YES
YES
YES
NO
NO
NO
NO
YES
YES
YES
YES
YES
YES
YES
YES
YES
YES
YES
YES
YES
YES
YES
YES
YES
YES
YES
YES
YES
YES
YES
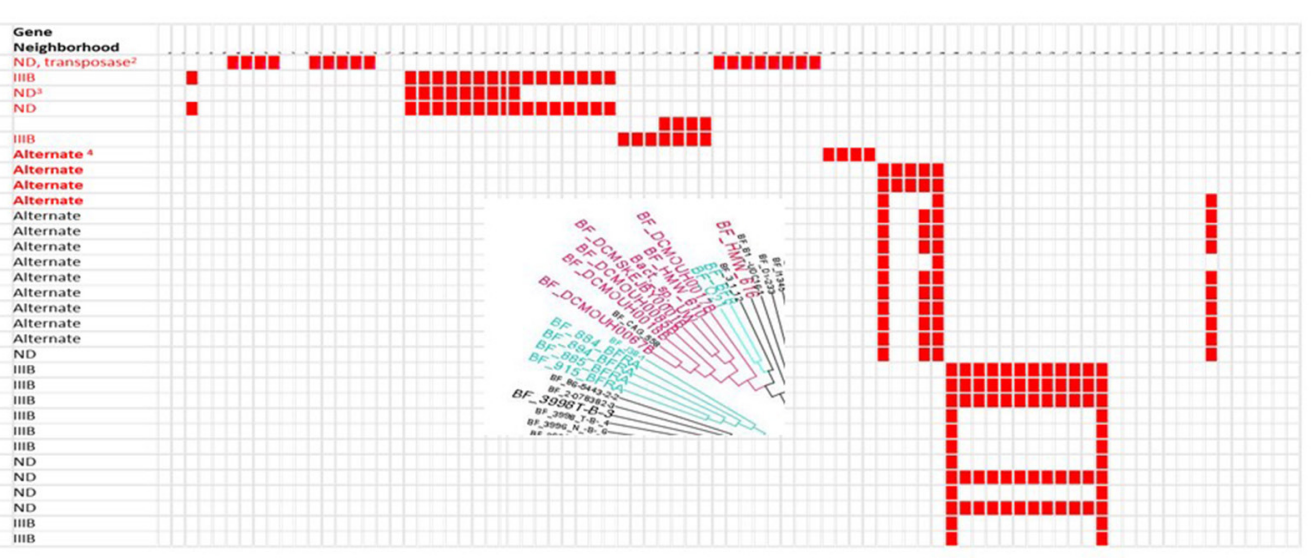

${ }^{1}$ Probably same CRISPR found on different contig

${ }^{2} \mathrm{ND}$, contig cut off but gene neighborhood is homologous to that of Type IIIB

${ }^{3}$ ND Repeat on contig by itself, adjacent genes not determined

${ }^{4}$ Alternate gene neighborhood in the midst of polysaccharide and other metabolic genes

${ }^{5} \mathrm{RT}$ Cas 1 not annotated but CMR4 is present

B $\mid$ BF-S13-L11-

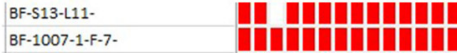

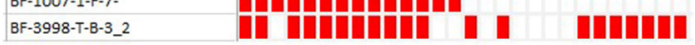

C

BF-3774-T13BF-DCMOUHOOA28.

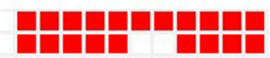

FIGURE 7 | Binary representation of spacer distribution in Type IIIB CRISPR-Cas in blood isolates and other strains with repeat arrays in alternate neighborhoods. The CRISPRs are configured with the oldest spacer at the "top" of the array (i.e., spacer 1, to the left edge of the binary representation) thus the newest spacers are those at the right edge. (A) This is an excerpted panel of the full binary representation of spacer distribution in Supplementary Table 3B. Each unique spacer is represented by a red vertical bar. Isolates from blood are in a red font. A unique pattern of spacers are found in the atypical neighborhood. Four of the blood isolates have no other Type IIIB CRISPR-Cas array and no Type IIIB cas genes. There are no cas genes adjacent to the CRISPR array in the atypical gene neighborhood, but since there is also a Type IIIB CRISPR-Cas array in the typical neighborhood, it is possible that the genes can act in trans on the atypical array. This is not the case for the four blood isolates (in bold red font). Blood isolates Bacteroides sp. UW and BF-DCMSKEJBY18 have two additional spacers not seen in other isolates but their protospacer targets could not be determined. A cutout of the dendrogram shown in Figure $\mathbf{1}$ is superimposed to show the phylogenetic relationship of the blood isolates. (B) Type IB spacer distribution in three strains of BF. BF-S13-L11 and BF-1007_1_F-7 are closely related phylogenetically while BF-3998_T-B-3_2 is at the opposite end of the phylogenetic tree; the more ancient part of the CRISPR (i.e., the first spacers) is highly conserved. BF-3998-T-B-3_2 has a longer array with more spacers at the newest edge, indicating that BF-3998-T-B-3_2 acquired more spacers. (C) Type IIIB spacer arrangements in two closely related strains of BF (see Figure 1). This pattern is consistent with the frequently seen homology of CRISPR arrays between highly related strains, with one strain losing two of the internal spacers. Another possible but less likely scenario is that BF-3774_T13 picked up two additional spacers that were not added at the leading edge but at an internal location. 
neighborhood with adjacent cas genes; thus, it is possible that the genes can act in trans on the atypical array. Again, this is not the case for isolates Bacteroides sp. UW, BF-DCMSKEJBY18, BF HMW 610, and BF-DCMOUH0067B. Blood isolates Bacteroides sp. UW and BF-DCMSKEJBY18 have two additional spacers not seen in other isolates. It is not clear how these atypical systems appeared but it is tempting to speculate that the atypical array broke away from the original CRISPR-Cas array and that the very virulent blood isolates somehow discarded the cas genes portion of the original array. At this point we have no evidence for that speculation nor a reason that it would be beneficial for those isolates to discard that array or adjacent genes. Figures $\mathbf{7 B}$ and $\mathbf{C}$ illustrate examples of spacer pattern diversions between closely related strains and spacer conservation between distant strains. Figure 7B shows distribution of two closely related strains and one distant strain and Figure 7C shows Type IIIB spacer arrangements in two closely related strains of $\mathrm{BF}$ and is consistent with a pattern in which spacers can get lost.

\section{The Plasticity of the B. fragilis Genome Is Balanced by Multiple Systems to Avoid Invading DNA Elements}

The B. fragilis genome is very "plastic," due both to its ability to incorporate pathogenicity islands from other $B$. fragilis and foreign genes via HGT as well as its ability to simply turn specific genes on or off as needed. Combined, these traits allow $B$. fragilis to adapt to new nutrition pathways, utilize specific efflux pumps to rid the cell of toxic substrates, and display new surface epitopes-taken together, allowing them to change from friendly commensal to dangerous threat (Wexler, 2007). We previously demonstrated transfer of a mobile element that contained multiple resistance genes of aerobic origin clustered within a conjugative transposon (Husain et al., 2014).

This ability of $B$. fragilis to easily incorporate foreign genes is intriguing since $B$. fragilis also possesses strong DNA restriction modification (DNA/RM) systems to degrade "nonself” DNA. We previously demonstrated horizontal transfer of mobile elements bearing alternate variants of these genes from a multidrug resistant $B$. fragilis isolate (HMW 615) to $B$. fragilis 638R (Husain et al., 2017). These systems are located in shufflons with invertible promoters that can be turned off or on, so that the bacterium can control whether the incoming DNA will be degraded (Patrick et al., 2010). If the system is turned "off," the incoming DNA can survive degradation. External or internal signals that regulate these systems have not yet been described. Now we have shown that in addition to the DNA/RM systems, there are abundant CRISPR elements in $B$. fragilis with adjacent cas genes, suggesting that these CRISPRs are indeed active as a bacterial defense system. We had previously suggested that predation by bacteriophages is an evolutionary driving force for generation of variable polysaccharide and R-M systems in B. fragilis (Patrick et al., 2010) and that this system can be diversified by HGT (Husain et al., 2017). In an interesting twist, B. fragilis also has a Type IIC CRISPR system with a high proportion of crRNAs with homology to $B$. fragilis phages; it is likely that this CRISPR system is largely directed to protect against invading bacteriophage (but these systems are lacking in the most virulent $B$. fragilis strains).

Thus, at least two unique systems are in place in $B$. fragilis to control and degrade incoming DNA, despite the well-established role of B. fragilis as a "resistance reservoir" (Salyers et al., 2004; Coyne et al., 2014) and its known ability to incorporate both other B. fragilis elements as well as "foreign" genes (Husain et al., 2014). It seems likely that an intricate balance of these two evolutionary forces is responsible for the remarkable adaptability of $B$. fragilis to the changing nutritional availability, immune forces and competitive organisms in the complex environment of the gut microbiome.

\section{CONCLUSIONS}

In this study, we identified and described CRISPR-Cas systems in $B$. fragilis as a first step for further exploration of the roles and mode of function of $B$. fragilis CRISPRs. We now need to determine whether the $B$. fragilis CRISPRs are functional in bacterial immunity and/or in regulation and whether they can signal acquisition of virulence determinants. Aside from these associations, there is growing evidence that CRISPR-Cas systems may have alternative roles that allow the bacterium to survive host defenses and replicate (Sampson and Weiss, 2014). For example, the Cas1 protein of the CRISPR-Cas system of E. coli may play a role in DNA repair (Babu et al., 2011). Since DNA damage to bacteria can be the result of specific host defenses during infection (e.g., the production of radical nitrogen and oxygen species), it would be beneficial to the bacterium to possess redundancy in its DNA repair capability (Sampson and Weiss, 2014). An additional way to avoid or repair DNA damage due to radical oxygen species would obviously be of great benefit to the anaerobic $B$. fragilis as well. In addition to these functions, there are reports of CRISPR-Cas involvement in resistance to stress, pathogenicity and regulation of biofilm formation (Barrangou, 2015).

We found that the most virulent strains of $B$. fragilis, the blood isolates, did not have Type IIC CRISPR-Cas systems, which may suggest that they remain capable of incorporating resistance and virulence factors that may be transferred via phages. Also, they do not appear to have functional Type IIIB systems, although they retain the CRISPR repeat array characteristic of that system, but in a distinct gene neighborhood. Thus, it appears that for these most virulent blood isolates, there is a benefit to being able to acquire genes from phages and possibly other mobile elements, which is completely consistent with the evidence that many antimicrobial resistance genes and other virulence genes are mobilized via HGT. Further analysis of the spacer acquisition pattern will help to determine how these spacers were acquired and help to elucidate the extent to which strains evolve by vertical evolution and/or horizontal gene transmission. Experimental molecular manipulation to determine the functions of the various CRISPR arrays will lead to a more comprehensive understanding of their functions in bacterial defense, gene 
regulation, virulence and commensalism of this important gut pathobiont.

\section{AUTHOR CONTRIBUTIONS}

MT contributed to the design, performed bioinformatic analyses, assisted in the interpretation of results and in writing the manuscript. HW designed the study, interpreted the results and wrote the manuscript. All authors have read and approved the final version of the manuscript.

\section{FUNDING}

These studies were supported by grants to $\mathrm{HW}$ from the Department of Veteran's Affairs (BX000563-01A1) and the National Institutes of Health (R21 AI109545-01).

\section{REFERENCES}

Alkhnbashi, O. S., Shah, S. A., Garrett, R. A., Saunders, S. J., Costa, F., and Backofen, R. (2016). Characterizing leader sequences of CRISPR loci. Bioinformatics 32, i576-i585. doi: 10.1093/bioinformatics/btw454

Ank, N., Sydenham, T. V., Iversen, L. H., Justesen, U. S., and Wang, M. (2015). Characterisation of a multidrug-resistant Bacteroides fragilis isolate recovered from blood of a patient in Denmark using whole-genome sequencing. Int. J. Antimicrob. Agents 46, 117-120. doi: 10.1016/j.ijantimicag.2015.02.024

Aziz, R., Bartels, D., Best, A., DeJongh, M., Disz, T., Edwards, R., et al. (2008). The RAST Server: rapid annotations using subsystems technology. BMC Genomics 9:75. doi: 10.1186/1471-2164-9-75

Babu, M., Beloglazova, N., Flick, R., Graham, C., Skarina, T., Nocek, B., et al. (2011). A dual function of the CRISPR-Cas system in bacterial antivirus immunity and DNA repair. Mol. Microbiol. 79, 484-502. doi: 10.1111/j.1365-2958.2010.07465.x

Barlow, M. (2009). What antimicrobial resistance has taught us about horizontal gene transfer. Methods Mol. Biol. 532, 397-411. doi: 10.1007/978-1-60327-853-9_23

Barrangou, R. (2015). Diversity of CRISPR-Cas immune systems and molecular machines. Genome Biol. 16, 247. doi: 10.1186/s13059-015-0816-9

Barrangou, R., Fremaux, C., Deveau, H., Richards, M., Boyaval, P., Moineau, S., et al. (2007). CRISPR provides acquired resistance against viruses in prokaryotes. Science 315, 1709-1712. doi: 10.1126/science.1138140

Bayley, D. P., Rocha, E. R., and Smith, C. J. (2000). Analysis of cepA and other Bacteroides fragilis genes reveals a unique promoter structure. FEMS Microbiol. Lett. 193, 149-154. doi: 10.1111/j.1574-6968.2000.tb09417.x

Benson, D. A., Clark, K., Karsch-Mizrachi, I., Lipman, D. J., Ostell, J., and Sayers, E. W. (2014). GenBank. Nucleic Acids Res. 42, D32-D37. doi: 10.1093/nar/gkt1030

Bland, C., Ramsey, T. L., Sabree, F., Lowe, M., Brown, K., Kyrpides, N. C., et al. (2007). CRISPR recognition tool (CRT): a tool for automatic detection of clustered regularly interspaced palindromic repeats. BMC Bioinformatics 8:209. doi: 10.1186/1471-2105-8-209

Bourgogne, A., Garsin, D. A., Qin, X., Singh, K. V., Sillanpaa, J., Yerrapragada, S., et al. (2008). Large scale variation in Enterococcus faecalis illustrated by the genome analysis of strain OG1RF. Genome Biol. 9:R110. doi: $10.1186 / \mathrm{gb}-2008-9-7-\mathrm{r} 110$

Burstein, D., Sun, C. L., Brown, C. T., Sharon, I., Anantharaman, K., Probst, A. J., et al. (2016). Major bacterial lineages are essentially devoid of CRISPR-Cas viral defence systems. Nat. Commun. 7:10613. doi: 10.1038/ncomms10613

Capozzi, V., and Spano, G. (2009). Horizontal gene transfer in the gut: is it a risk? Food Res. Int. 42, 1501-1502. doi: 10.1016/j.foodres.2009. 08.001

Carte, J., Christopher, R. T., Smith, J. T., Olson, S., Barrangou, R., Moineau, S., et al. (2014). The three major types of CRISPR-Cas systems function independently

\section{ACKNOWLEDGMENTS}

We wish to thank and acknowledge the kind assistance of Dr. Michael Richter (Ribocon, Bremen, Germany) in constructing a matrix of average nucleotide identity between two organisms (ANIb) values using the J species Web Server and of Dr. Luis M. Rodriguez (Kostas Lab, Georgia Institute of Technology, Atlanta, Georgia) in using an $\mathrm{R}$ routine to convert this data into a Newick tree file.

\section{SUPPLEMENTARY MATERIAL}

The Supplementary Material for this article can be found online at: https://www.frontiersin.org/articles/10.3389/fmicb. 2017.02234/full\#supplementary-material

in CRISPR RNA biogenesis in Streptococcus thermophilus. Mol. Microbiol. 93, 98-112. doi: 10.1111/mmi.12644

Chénard, C., Wirth, J. F., and Suttle, C. A. (2016). Viruses infecting a freshwater filamentous Cyanobacterium (Nostoc sp.) encode a functional CRISPR array and a proteobacterial DNA polymerase B. MBio 7:e00667-16. doi: 10.1128/mBio.00667-16

Chylinski, K., Le Rhun, A., and Charpentier, E. (2013). The tracrRNA and Cas9 families of type II CRISPR-Cas immunity systems. RNA Biol. 10, 726-737. doi: 10.4161/rna.24321

Chylinski, K., Makarova, K. S., Charpentier, E., and Koonin, E. V. (2014). Classification and evolution of type II CRISPR-Cas systems. Nucleic Acids Res. 42, 6091-6105. doi: 10.1093/nar/gku241

Correia, F. F., D’Onofrio, A., Rejtar, T., Li, L., Karger, B. L., Makarova, K., et al. (2006). Kinase activity of overexpressed HipA is required for growth arrest and multidrug tolerance in Escherichia coli. J. Bacteriol. 188, 8360-8367. doi: 10.1128/JB.01237-06

Coyne, M. J., Zitomersky, N. L., McGuire, A. M., Earl, A. M., and Comstock, L. E. (2014). Evidence of extensive DNA transfer between Bacteroidales species within the human gut. MBio 5, e01305-e01314. doi: 10.1128/mBio.01305-14

Crooks, G. E., Hon, G., Chandonia, J. M., and Brenner, S. E. (2004). WebLogo: a sequence logo generator. Genome Res. 14, 1188-1190. doi: 10.1101/gr.849004

Day, B. J., van Heeckeren, A. M., Min, E., and Velsor, L. W. (2004). Role for cystic fibrosis transmembrane conductance regulator protein in a glutathione response to bronchopulmonary pseudomonas infection. Infect. Immun. 72, 2045-2051. doi: 10.1128/IAI.72.4.2045-2051.2004

Ding, Y., Chan, C. Y., and Lawrence, C. E. (2005). RNA secondary structure prediction by centroids in a Boltzmann weighted ensemble. RNA 11, 1157-1166. doi: 10.1261/rna.2500605

Godde, J. S., and Bickerton, A. (2006). The repetitive DNA elements called CRISPRs and their associated genes: evidence of horizontal transfer among prokaryotes. J. Mol. Evol. 62, 718-729. doi: 10.1007/s00239-005-0223-z

Grissa, I., Vergnaud, G., and Pourcel, C. (2007). CRISPRFinder: a web tool to identify clustered regularly interspaced short palindromic repeats. Nucleic Acids Res. 35, W52-W57. doi: 10.1093/nar/gkm360

Haft, D. H., Selengut, J., Mongodin, E. F., and Nelson, K. E. (2005). A guild of 45 CRISPR-associated (Cas) protein families and multiple CRISPR/Cas subtypes exist in prokaryotic genomes. PLoS Comput. Biol. 1:e60. doi: 10.1371/journal.pcbi.0010060

Han, M. V., and Zmasek, C. M. (2009). phyloXML: XML for evolutionary biology and comparative genomics. BMC Bioinformatics 10:356. doi: 10.1186/1471-2105-10-356

Hansen, S., Vulić, M., Min, J., Yen, T. J., Schumacher, M. A., Brennan, R. G., et al. (2012). Regulation of the Escherichia coli HipBA toxin-antitoxin system by proteolysis. PLoS ONE 7:e39185. doi: 10.1371/annotation/e608601c-eadd-4c11-adb2-7b605aba9c44 
Heler, R., Samai, P., Modell, J. W., Weiner, C., Goldberg, G. W., Bikard, D., et al. (2015). Cas9 specifies functional viral targets during CRISPR-Cas adaptation. Nature 519, 199-202. doi: 10.1038/nature14245

Husain, F., Tang, K., Yaligara, V., Boente, R., Blakely, G., Patrick, S., et al. (2017). Novel large-scale chromosomal transfer in Bacteroides fragilis contributes to its pan-genome and rapid environmental adaptation. Microb. Genom. 4:e000136. doi: 10.1099/mgen.0.000136

Husain, F., Veeranagouda, Y., Boente, R., Tang, K., Mulato, G., and Wexler, H. M. (2014). The Ellis Island effect: a novel mobile element in a multi-drug resistant Bacteroides fragilis clinical isolate includes a mosaic of resistance genes from Gram-positive bacteria. Mob. Genet. Elem. 4, E29801-E29812. doi: $10.4161 /$ mge. 29801

Iacobino, A., Scalfaro, C., and Franciosa, G. (2013). Structure and genetic content of the megaplasmids of neurotoxigenic Clostridium butyricum type E strains from Italy. PLoS ONE 8:e71324. doi: 10.1371/journal.pone.0071324

Jiang, W., Samai, P., and Marraffini, L. A. (2016). Degradation of phage transcripts by CRISPR-associated RNases enables type III CRISPR-Cas immunity. Cell 164, 710-721. doi: 10.1016/j.cell.2015.12.053

Jore, M. M., Lundgren, M., van Duijn, E., Bultema, J. B., Westra, E. R., Waghmare, S. P., et al. (2011). Structural basis for CRISPR RNA-guided DNA recognition by Cascade. Nat. Struct. Mol. Biol. 18, 529-536. doi: 10.1038/nsmb.2019

Kalapila, A., Pergam, S., Pottinger, P., Butler-Wu, S., Whimbey, E., and Duchin, J. (2013). Multidrug-resistant Bacteroides fragilis-Seattle, Washington, 2013. MMWR Morb. Mortal. Wkly. Rep. 62, 694-696.

Kau, A. L., Planer, J. D., Liu, J., Rao, S., Yatsunenko, T., Trehan, I., et al. (2015). Functional characterization of IgA-targeted bacterial taxa from undernourished Malawian children that produce diet-dependent enteropathy. Sci. Transl. Med. 7:276ra224. doi: 10.1126/scitranslmed.aaa4877

Kunin, V., Sorek, R., and Hugenholtz, P. (2007). Evolutionary conservation of sequence and secondary structures in CRISPR repeats. Genome Biol. 8:R61. doi: 10.1186/gb-2007-8-4-r61

Kurokawa, K., Itoh, T., Kuwahara, T., Oshima, K., Toh, H., Toyoda, A., et al. (2007). Comparative metagenomics revealed commonly enriched gene sets in human gut microbiomes. DNA Res. 14, 169-181. doi: 10.1093/dnares/dsm018

Kuwahara, T., Yamashita, A., Hirakawa, H., Nakayama, H., Toh, H., Okada, N., et al. (2004). Genomic analysis of Bacteroides fragilis reveals extensive DNA inversions regulating cell surface adaptation. Proc. Natl. Acad. Sci. U.S.A. 101, 14919-14924. doi: 10.1073/pnas.0404172101

Lange, S. J., Alkhnbashi, O. S., Rose, D., Will, S., and Backofen, R. (2013). CRISPRmap: an automated classification of repeat conservation in prokaryotic adaptive immune systems. Nucleic Acids Res. 41, 8034-8044. doi: $10.1093 /$ nar/gkt606

Louwen, R., Staals, R. H., Endtz, H. P., van Baarlen, P., and van der Oost, J. (2014). The role of CRISPR-Cas systems in virulence of pathogenic bacteria. Microbiol. Mol. Biol. Rev. 78, 74-88. doi: 10.1128/MMBR.00039-13

Makarova, K. S., Grishin, N. V., Shabalina, S. A., Wolf, Y. I., and Koonin, E. V. (2006). A putative RNA-interference-based immune system in prokaryotes: computational analysis of the predicted enzymatic machinery, functional analogies with eukaryotic RNAi, and hypothetical mechanisms of action. Biol. Direct 1:7. doi: 10.1186/1745-6150-1-7

Makarova, K. S., Haft, D. H., Barrangou, R., Brouns, S. J., Charpentier, E., Horvath, P., et al. (2011). Evolution and classification of the CRISPR-Cas systems. Nat. Rev. Microbiol. 9, 467-477. doi: 10.1038/nrmicro2577

Makarova, K. S., Wolf, Y. I., Alkhnbashi, O. S., Costa, F., Shah, S. A., Saunders, S. J., et al. (2015). An updated evolutionary classification of CRISPR-Cas systems. Nat. Rev. Microbiol. 13, 722-736. doi: 10.1038/nrmicro3569

Marinelli, L. J., Fitz-Gibbon, S., Hayes, C., Bowman, C., Inkeles, M., Loncaric, A., et al. (2012). Propionibacterium acnes bacteriophages display limited genetic diversity and broad killing activity against bacterial skin isolates. MBio 3:12. doi: 10.1128/mBio.00279-12

Markowitz, V., Chen, I. M., Palaniappan, K., Chu, K., Szeto, E., Grechkin, Y., et al. (2012). IMG: the Integrated Microbial Genomes database and comparative analysis system. Nucleic Acids Res. 40, 22. doi: 10.1093/nar/gkr1044

Marraffini, L. A. (2015). CRISPR-Cas immunity in prokaryotes. Nature 526, 55-61. doi: 10.1038/nature15386

Mimee, M., Tucker, A. C., Voigt, C. A., and Lu, T. K. (2015). Programming a human commensal bacterium, bacteroides thetaiotaomicron, to sense and respond to stimuli in the murine gut microbiota. Cell Syst. 1, 62-71. doi: 10.1016/j.cels.2015.06.001
Mougiakos, I., Bosma, E. F., de Vos, W. M., van Kranenburg, R., and van der Oost, J. (2016). Next generation prokaryotic engineering: the CRISPR-Cas toolkit. Trends Biotechnol. 34, 575-587. doi: 10.1016/j.tibtech.2016.02.004

Nikitina, A. S., Kharlampieva, D. D., Babenko, V. V., Shirokov, D. A., Vakhitova, M. T., Manolov, A. I., et al. (2015). Complete genome sequence of an enterotoxigenic Bacteroides fragilis clinical isolate. Genome Announc. 3:e00450-15. doi: 10.1128/genomeA.00450-15

Nuñez, J. K., Lee, A. S., Engelman, A., and Doudna, J. A. (2015). Integrasemediated spacer acquisition during CRISPR-Cas adaptive immunity. Nature 519, 193-198. doi: 10.1038/nature14237

Palmer, K. L., and Gilmore, M. S. (2010). Multidrug-resistant enterococci lack CRISPR-cas. MBio 1:10. doi: 10.1128/mBio.00227-10

Patrick, S., Blakely, G. W., Houston, S., Moore, J., Abratt, V. R., Bertalan, M., et al. (2010). Twenty-eight divergent polysaccharide loci specifying withinand amongst-strain capsule diversity in three strains of Bacteroides fragilis. Microbiology 156(Pt 11), 3255-3269. doi: 10.1099/mic.0.042978-0

Pierce, J. V., and Bernstein, H. D. (2016). Genomic diversity of enterotoxigenic strains of Bacteroides fragilis. PLoS ONE 11:e0158171. doi: 10.1371/journal.pone.0158171

Privitera, G. F., Dublanchet, A. F., and Sebald, M. (1979). Transfer of multiple antibiotic resistance between subspecies of Bacteroides fragilis. J. Infect. Dis. 139, 97-101. doi: 10.1093/infdis/139.1.97

Pumbwe, L., Chang, A., Smith, R. L., and Wexler, H. M. (2007a). BmeRABC5 is a multidrug efflux system that can confer metronidazole resistance in Bacteroides fragilis. Microb. Drug Resist. 13, 96-101. doi: 10.1089/mdr.2007.719

Pumbwe, L., Wareham, D. W., Aduse-Opoku, J., Brazier, J. S., and Wexler, H. M. (2007b). Genetic analysis of mechanisms of multidrug resistance in a clinical isolate of Bacteroides fragilis. Clin. Microbiol. Infect. 13, 183-189. doi: 10.1111/j.1469-0691.2006.01620.x

Rath, D., Amlinger, L., Rath, A., and Lundgren, M. (2015). The CRISPRCas immune system: biology, mechanisms and applications. Biochimie 117, 119-128. doi: 10.1016/j.biochi.2015.03.025

Risse, J., Thomson, M., Patrick, S., Blakely, G., Koutsovoulos, G., Blaxter, M., et al. (2015). A single chromosome assembly of Bacteroides fragilis strain BE1 from Illumina and MinION nanopore sequencing data. Gigascience 4, 60. doi: 10.1186/s13742-015-0101-6

Roach, D. J., Burton, J. J., Lee, C., Stackhouse, B., Butler-Wu, S. M., Cookson, B. T., et al. (2015). A year of infection in the intensive care unit: prospective whole genome sequencing of bacterial clinical isolates reveals cryptic transmissions and novel microbiota. PLoS Genet. 11:e1005413. doi: 10.1371/journal.pgen.1005413

Salipante, S. J., Kalapila, A., Pottinger, P. S., Hoogestraat, D. R., Cummings, L., Duchin, J. S., et al. (2015). Characterization of a multidrugresistant, novel bacteroides genomospecies. Emerg. Infect. Dis. 21, 95-98. doi: 10.3201/eid2101.140662

Salyers, A. A., Gupta, A., and Wang, Y. (2004). Human intestinal bacteria as reservoirs for antibiotic resistance genes. Trends Microbiol. 12, 412-416. doi: 10.1016/j.tim.2004.07.004

Sampson, T., and Weiss, D. (2014). CRISPR-Cas systems: new players in gene regulation and bacterial physiology. Front. Cell. Infect. Microbiol. 4:37. doi: $10.3389 /$ fcimb.2014.00037

Sander, J. D., and Joung, J. K. (2014). CRISPR-Cas systems for editing, regulating and targeting genomes. Nat. Biotechnol. 32, 347-355. doi: 10.1038/ nbt. 2842

Schapiro, J. M., Gupta, R., Stefansson, E., Fang, F. C., and Limaye, A. P. (2004). Isolation of metronidazole-resistant Bacteroides fragilis carrying the nimA nitroreductase gene from a patient in Washington State. J. Clin. Microbiol. 42, 4127-4129. doi: 10.1128/JCM.42.9.4127-4129.2004

Schumacher, M. A., Balani, P., Min, J., Chinnam, N. B., Hansen, S., Vulić, M., et al. (2015). HipBA-promoter structures reveal the basis of heritable multidrug tolerance. Nature 524, 59-64. doi: 10.1038/nature14662

Science, U.o.M.S.o.M.I.f.G. (2013). GSCID Genomic Sequencing Center for Infectious Disease [Online]. NCBI. Available online at: http://gscid.igs. umaryland.edu/wp.php?wp=comparative_genomics_of_human_mucosal_ isolates_of_the_enterotoxigenic_bacteroides_fragilis_group.

Sherwood, J., Fraser, S., Citron, D., Wexler, H., Blakely, G., Jobling, K., et al. (2011). Multi-drug resistant Bacteroides fragilis recovered from blood and severe leg wounds caused by an improvised explosive device (IED) in Afghanistan. Anaerobe 17, 152-155. doi: 10.1016/j.anaerobe.2011.02.007 
Silas, S., Mohr, G., Sidote, D. J., Markham, L. M., Sanchez-Amat, A., Bhaya, D., et al. (2016). Direct CRISPR spacer acquisition from RNA by a natural reverse transcriptase-Cas1 fusion protein. Science 351:aad4234. doi: $10.1126 /$ science.aad 4234

Soki, J., Hedberg, M., Patrick, S., Balint, B., Herczeg, R., Nagy, I., et al. (2016). Emergence and evolution of an international cluster of MDR Bacteroides fragilis isolates. J. Antimicrob. Chemother. 71, 2441-2448. doi: 10.1093/jac/ dkw175

Solomkin, J. S., Mazuski, J. E., Bradley, J. S., Rodvold, K. A., Goldstein, E. J., Baron, E. J., et al. (2010). Diagnosis and management of complicated intra-abdominal infection in adults and children: guidelines by the Surgical Infection Society and the Infectious Diseases Society of America. Surg. Infect. (Larchmt) 11, 79-109. doi: 10.1089/sur.2009.9930

Sorek, R., Kunin, V., and Hugenholtz, P. (2008). CRISPR-a widespread system that provides acquired resistance against phages in bacteria and archaea. Nat. Rev. Microbiol. 6, 181-186. doi: 10.1038/nrmicrol793

Stern, A., Keren, L., Wurtzel, O., Amitai, G., and Sorek, R. (2010). Self-targeting by CRISPR: gene regulation or autoimmunity? Trends Genet. 26, 335-340. doi: 10.1016/j.tig.2010.05.008

Sydenham, T. V., Soki, J., Hasman, H., Wang, M., Justesen, U. S., and Esgai (2015). Identification of antimicrobial resistance genes in multidrug-resistant clinical Bacteroides fragilis isolates by whole genome shotgun sequencing. Anaerobe 31, 59-64. doi: 10.1016/j.anaerobe.2014.10.009
Wexler, H. M. (2007). Bacteroides-the good, the bad, and the nitty-gritty. Clin Microbiol. Rev. 20, 593-621. doi: 10.1128/CMR.00008-07

Wexler, H. M. (2014). "The genus Bacteroides," in The Prokaryotes: Other major lineages of Bacteria and the Archaea, eds E. Y. Rosenberg, E. F. DeLong, F. Thompson, S. Lory, and E. Stackebrandt (Berlin; Heidelberg: Springer Verlig), 459-484.

Zhu, D. K., Yang, X. Q., He, Y., Zhou, W. S., Song, X. H., Wang, J. B., et al. (2016). Comparative genomic analysis identifies structural features of CRISPR-Cas systems in Riemerella anatipestifer. BMC Genomics 17:689. doi: 10.1186/s12864-016-3040-4

Conflict of Interest Statement: The authors declare that the research was conducted in the absence of any commercial or financial relationships that could be construed as a potential conflict of interest.

The reviewer $\mathrm{AO}$ and handling Editor declared their shared affiliation.

Copyright (c) 2017 Tajkarimi and Wexler. This is an open-access article distributed under the terms of the Creative Commons Attribution License (CC BY). The use, distribution or reproduction in other forums is permitted, provided the original author(s) or licensor are credited and that the original publication in this journal is cited, in accordance with accepted academic practice. No use, distribution or reproduction is permitted which does not comply with these terms. 